HANNA JENNI

\title{
Diathese und Modus des ägyptischen Pseudopartizips
}

1. Das mediale Pseudopartizip des Altägyptischen

1.1 Die Rolle des Agens

1.2 Subjekt, Objekt und Kausativierung

1.3 Die Rolle des Benefaktivs

1.4 Zur Negierung des Pseudopartizips

2. Die angepasste Medialität des Pseudopartizips im Mittelägyptischen

2.1 Die angebliche Relikthaftigkeit des mittelägyptischen Pseudopartizips

2.2 Modalisierung

3. Exkurs: passive Konstruktion mit Angabe des Agens vs. aktive Konstruktion

4. Typologische Entwicklung und Semantik des Résumé ägyptischen Pseudopartizips

Summary

\section{Das mediale Pseudopartizip des Altägyptischen}

Das Pseudopartizip ist als ursprünglich mediale perfektive Form erkannt worden ${ }^{1}$. Das soll zunächst für das Altägyptische näher betrachtet werden.

\subsection{Die Rolle des Agens}

Ein Teil der Verwendung des Pseudopartizips ist bekanntlich intransitiv. Das Pseudopartizip hat generell perfektiven Aspekt und kann deontisch-modal interpretiert werden (Bsp. 2f.).

1. $m 3 n=f k w$ 3h.tj, ,Er soll dich sehen, indem du ein Geist bist ${ }^{\text {"6 }}$.

2. hntj $r=k r \breve{s} n m(j) r w 3 d-w r$ [...] 3h.tj $m$ 3h.t dd.tj $m$ $\underline{d} \underline{d} . t$, „Fahr stromaufwärts zum See, befahre das Meer [...] du sollst ein Geist sein / sei ein Geist und du sollst dauerhaft sein / sei dauerhaft in Mendes! : $^{3}$

3. $\underline{d} d m d w$ wr.tj N.N. pw $\underline{d} 3(j) . t j$ N.N. $p w$, „Zu rezitieren: Du sollst gross sein / Sei gross, N.N. da!

1 A. Loprieno, Ancient Egyptian, Cambridge 1995,$66 ; 83 \mathrm{f}$

${ }^{2}$ Pyr. 1618a (Spr. 592, M)

${ }^{3}$ Pyr. 1260b-1261b (Spr. 532, N).
Du sollst hinüberfahren / Fahre hinüber, N.N. da! ${ }^{4}$.

4. $j \cdot d(j) j w t=f j w=f w r b(w)$, „Lass ihn kommen, (denn) er ist rein ${ }^{45}$.

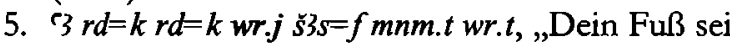
groß! Indem/weil dein $F u ß$ groß ist, wird er das große Bett (= Himmel) durchschreiten" ${ }^{\text {"6 }}$.

Eine weitere Verwendung des Pseudopartizips ist transitiv-passiv (zur aktiven Verwendung siehe Abschnitt 1.2).

6. $\underline{d} d m d w W$ Wjir N.N. nd.tj $r \underline{d}(j) . n(=j) n=k n \underline{t r} . w n b . w$, „Zu rezitieren: Osiris N.N., du sollst geschützt sein; ich gebe dir alle Götter" ${ }^{\text {“? }}$.

7. $j j n=k$ N.N. pw $m$ jwnw nd.tj, „Dieser N.N. kommt zu dir in Heliopolis, indem/so dass du geschützt bist" ${ }^{8}$.

In der intransitiv-aktiven Verwendung sind Verben involviert, die keine (semantische) Agensrolle vorgesehen haben (ohne agent-slot) und im folgenden nonagentivische Verben genannt sind, in der transitiv-passiven Verwendung sind dagegen agentivische Verben anzutreffen, die eine Agensrolle besetzen können. Es sind im allgemeinen die atelischen Verben, die nonagentivisch sind, und die telischen die agentivisch sind.

\begin{tabular}{|c|c|c|}
\hline Atelisch & & Telisch \\
\hline activity & achievement & accomplishment \\
\hline
\end{tabular}

Am nonagentivischen Ende der Skala stehen Verben wie $w d \underline{d}$, „heil sein“, am agentivischen Verben wie $w h 3$, ,ausgebrochen worden sein (Stein)“ oder kausatives shr, ,gefällt sein“.

${ }^{4}$ Pyr. 658a (Spr. 374, T).

${ }^{5}$ Pyr. 1141a (Spr. 510, P).

${ }^{6}$ Pyr. 658c (Spr. 374, T).

${ }^{7}$ Pyr. 775a (Spr. 425, P).

${ }^{8}$ Pyr. 2097c (Spr. 690, N). 
Im Falle eines Verbs wie $(w)^{r} b$, , (sich) waschen/reinigen", ist eine ganze Skala von Handlungen vorstellbar, die von agentivisch bis nonagentivisch reichen: Wasser und Holz Holen, Seife Besorgen, Feuer Machen und ein in den Schlamm gefallenes Kind Schrubben (ziemlich agentivisch! und operativ) oder sich kultisch Reinigen durch Hände in Wasserbecken Eintauchen und damit übers Gesicht Streichen (wenig agentivisch und operativ-faktitiv) oder einen heiligen Gegenstand Berühren und eine Gebetsformel Murmeln (kaum agentivisch und faktitiv) oder ohne Sünde Sein (nonagentivisch und stativisch) ${ }^{9}$. In dem Bereich, wo die Bedeutung von der Agentivität in die Nonagentivität kippt (oder, syntaktisch gesprochen, von der Transitivität in die Intransitivität), übernimmt das Pseudopartizip seine Funktion oder gibt sie, in umgekehrter Richtung gedacht und den Pfeilen in der oben gegebenen Darstellung entsprechend, ab.

8. $w^{r} b . w r=j n h t j b=s n r=s,,[\ldots]$ indem sie rein sind mir gegenüber und indem ihr Herz stark ist dabei" ${ }^{\prime 10}$.

9. $w^{c} b=s$ N.N. pn sntr $=s$ N.N. pn, ,Sie reinigt diesen N.N. (mit Wasser), sie reinigt diesen N.N. (mit Weihrauch) "“11.

Folgende Stellen aus den Pyramidentexten zeigen das Nebeneinander von Pseudopartizip und Imperativ mit identischem intransitiven, nonagentivischen Verb.

10. $d d m d w{ }^{\top} n h{ }^{\top} n h . t j j t(=j) m r n=k p w h r n t r w, ~, Z u$ rezitieren: Lebe und sei lebendig, mein Vater, (in diesem deinem Namen) wie du auch heißen magst, bei den Göttern! “'12.

11. ' $n h{ }^{\top}$ 'nh.tj $j t(=j) W_{s j r} p w\{m r n=k p w\} \quad h r j 3 h . w$, „Lebe und sei lebendig, mein Vater Osiris N.N. da, ((in diesem deinem Namen) wie du auch heißen magst, $\}$ bei den Geistern! " ${ }^{13}$.

12. ' $n h$ 'nh.tj $m p$ mp.tj jr $\underline{d} b^{e} j t=k j r d b^{e} s 3 h$ jr p.t, „Lebe und sei lebendig, sei jung und sei verjüngt

${ }^{2} \mathrm{Zu}$ „operativ" vs. „faktitiv" und Kombinationsmöglichkeit siehe John Lyons, Semantics, Cambridge etc. 1977 , Bd. 2, 491.

${ }^{10}$ E. Edel, Altägyptische Grammatik, 2 Bde (AnOr 34/39), Roma 1955-1964, 210, \465.

${ }^{11}$ Pyr. 1181b (Spr. 515, P).

${ }^{12}$ Pyr. 1724a (Spr. 611, M).

${ }^{13}$ Pyr. 1899a (Spr. 665, N). im Einflussbereich deines Vaters und des Orions am Himmel! ${ }^{\text {cit }}$.

13. $j n<k>n d k w n(j) d d . n(=j) n d k w$ ' $n h . t j{ }^{\prime} n h . t j$, ,Ich bin es, der dich schützt, ich zögere nicht, dich zu schützen, und so sollst du ganz lebendig sein"; ' $n h n(j) t w \mid I I I I I I / / w d 3 . t j r=s n$, „lebe, denn zu dir gehörig IIIIIIIIII, so dass du heiler bist als sie"; ¿nh $r=f j t(=j) W_{s j r} N . N . d(j)(=j) n=k j r . t \quad H r h r=k$, „Lebe doch, mein Vater Osiris N.N., denn ich habe für dich das Horusauge an dir befestigt" (Ende des Spruches) ${ }^{15}$.

Die Doppelung zeigt die bekannten verschiedenen Aspekte der Verbformen, nämlich den nichtmarkierten beim Imperativ und beim Pseudopartizip den perfektiven. Darüber hinaus ist eine aktivere Nuance auf der einen und eine medial-passivere auf der anderen Seite auszumachen: eine angestrengtere Variante im Falle des Imperativs und der prospektiven $s \underline{d} m=f$-Form und eine entspanntere in der Obhut göttlicher Wesen im Falle des Pseudopartizips. Es ist kein Zufall, dass die Pseudopartizipien näher bei den göttlichen Größen stehen, die dem Verstorbenen gegenüber sozusagen die Agentivität abnehmen - die Agentivität, die im Pseudopartizip marginalisiert ist (Bsp.10-12). In Bsp. 13a-b, ist das entspannte Leben die Folge der göttlichen Fürsorge und das entspannte Heilsein die Folge einer bestimmten (Zerstörung im Text) Zugehörigkeit $^{16}$. Der Imperativ $n h$ im dritten Teil des Zitats ist mit einem aufmunternden $r=f$ versehen, das den Verbalinhalt verstärkt ${ }^{17}$.

Das altägyptische Pseudopartizip eignet sich wegen der semantischen Marginalisierung des Agens zur unpersönlichen Verwendung (vgl. auch Bsp. 46) und kann so auch als eingebettetes Objekt verwendet werden: $[\mathrm{A}]_{\mathrm{S}}[\mathrm{tut}]_{\mathrm{P}}$ [es muss

${ }^{14}$ Pyr. 2180b-c (Spr. 699, N).

${ }^{15}$ Pyr. 1797b-c (Spr. 636, N).

${ }^{16}$ Die Wortfolge 'nh.tj ${ }^{\prime}$ hh.tj ${ }^{\prime} n h$ am Übergang von Bsp. 13a zu 13b kann nicht als Einheit gelesen werden; so R. O. Faulkner, The Ancient Egyptian Pyramid Texts, Oxford 1969, 263: ,it is I who protect you and I will never fail to protect you. Live, live [Anm.: Hortative old perfectives + ' $n h$ as a noun:, live. Live the life'], because you are $[. .$.$] , you being more hale than they.$ Live, O my father Osiris the King, for I set for you the Eye of Horus on you“.

${ }^{17} \mathrm{Vgl}$. H. Jenni, Die pronominalen Erweiterungen beim Imperativ und der Ausdruck verbaler Reflexivität im Ägyptischen, in: ZÄS 132, 2005, 119 f. 
getan werden $]_{O}=$ Pseudoparizip $\rightarrow \mathrm{A}$ tut, was getan werden muss.

14. $s d r j r(j) . t j r(j) . t j w d . t w d . t j$, „Es schläft diejenige, die tut, was getan werden muss/zu tun ist, und die befiehlt, was befohlen werden muss/zu befehlen ist ${ }^{66^{18}}$.

Die Angabe des Agens bei einem transitivpassiven Pseudopartizip durch die Partikel $j n$ ist auffallend selten (vgl. Bsp. 15 mit 16 und 17 mit 18) oder aber es wird eine Konstruktion mit $w$-Passiv oder $t w$-Passiv gewählt (vgl. Bsp. $19 \mathrm{f}$. mit 21f.; siehe auch Abschnitt 3).

15. $h s(j) . k(w j) h r=s j n n b=j$, ,Ich wurde ausgezeichnet deswegen von meinem Herrn ${ }^{\prime \prime 19}$.

16. $h s(j) . k(w j) h r=s$ ' 3 , „Ich wurde sehr ausgezeichnet deswegen ${ }^{20}$.

17. $j w=j h 3 b . k(w j) r h w . t-n b w$ jn $h 3 t[j-r / / / /$, ,ICh wurde nach Hatnub ausgesandt durch den Hatia $/ / /^{421}$.

18. $j w=j h 3 b . k(w j) r h w . t-n b w$ tn $r j r(j) . t k 3 . t n h 3 t j-r$ [...], „Ich wurde ausgesandt nach diesem Hatnub, um einen Auftrag auszuführen für den Hatia $[\ldots]^{c^{22}}$.

19. $j r(j . w) n=f n m . t s j$ j.s $3 h(. w)$ jn $w t(j)$, ,Möge für ihn die (Zeremonie der) Überfahrt über den See ausgeführt werden, indem/nachdem er zu einem Geist gemacht worden ist durch den Balsamierungspriester ${ }^{<23}$.

20. $\underline{d} d \quad m d w t 3 \quad k 3(. w) h r r n w . t$ jn $\ulcorner. w j=t$, Tfn.t, , $\mathrm{Zu}$ rezitieren: Die Erde ist hochgehoben unter dem Himmel durch dein Armpaar, Tefnut ${ }^{c^{24}}$.

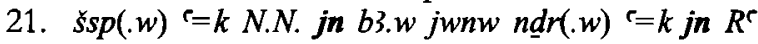
$t s(j . w) t p=k$ N.N. jn psd.tj [...], „Dein Arm werde ergriffen, N.N., durch die Mächte von Heliopolis, dein Arm werde gepackt durch Re, dein Kopf werde erhoben, N.N., durch die beiden Neunheiten $[\ldots]^{4{ }^{25}}$.

22. $n(j)$ snj.w N.N. pn jn $t 3 n(j)$ šnj.w (j)h.t-wt.t jn $t 3$, "Wird dieser N.N. nicht durch die Erde verflucht, so wird die (j)h.t-wt.t-Schlange nicht von der Erde verflucht ${ }^{، 26}$.

\footnotetext{
${ }^{18}$ Pyr. 1099b (Spr. 506, M).

${ }^{19}$ Urk. I, 255, 5.

${ }^{20}$ Urk. I, 137, 6.

${ }^{21} \mathrm{R}$. Anthes, Die Felsinschriften von Hatnub nach den Aufnahmen Georg Möllers (Untersuchungen zur Geschichte und Altertumskunde Aegyptens, Bd. 9), Hildesheim 1964, Taf. 11, Gr. 6, Kol. 4.

${ }^{22}$ Anthes, Felsinschriften (wie Anm. 21), Taf. 13, Gr. 9, Kol. 7.

${ }^{23}$ Urk. I, 189, 18.

${ }^{24}$ Pyr. 1405a (Spr. 562, P).

${ }^{25}$ Pyr. 1261b-1262b (Spr. 532, N).

${ }^{26}$ Pyr. 2110a-b (Spr. 690, N).
}

Wenn das Agens durch die Partikel jn eingeführt wird, erfährt es semantische Marginalisierung; morphosyntaktisch gesehen wird es extra, außerhalb der Verbalform, markiert. Semantisch gesehen handelt es sich bei den mit einem Pseudopartizip verwendeten Größen in der Rolle des Agens (Bsp. 15, 17, 19f.) um solche, die ko(n)textuell nicht neu sind resp. in der Hierarchie der salience weit oben stehen $(1 .>2$. $>$ 3. Person $>$ Eigenname $>$ menschlich $>$ belebt $>$ unbelebt, von oben nach unten). In Bsp. 15, 17 und 19 geht es um Handlungen, die nach den kulturellen Gegebenheiten von niemandem anderen als von dem entsprechenden Beamten oder Herrn (Auszeichnung, Aussendung des Beamten) oder dem entsprechenden Priester (Ritualhandlung) vorgenommen werden (können). Bsp. 16 und 18 verzichten ganz auf diese Angabe, weil das Agens selbstverständlich, d. h. aus der Erfahrungswelt des Adressaten ableitbar, oder dem Kontext zu entnehmen ist. In Bsp. 20 ist das Agens der mit Possessivpronomen versehene Körperteil der angeredeten Gottheit und kommt so einem Pronomen gleich (,durch dich, Tefnut"). Pronomina stehen in der Hierarchie der salience zuoberst, egal ob kataphorisch (wie hier) oder anaphorisch verwendet. Dagegen handelt es sich bei den als Agens beteiligten Größen in Bsp. 21f. mit w-Passiven um kontextuell neue Größen. (Vgl. auch Abschnitt 2.2 mit Bsp. 80 und Abschnitt 3.)

\subsection{Subjekt, Objekt und Kausativierung}

Während in den Pyramidentexten die 1. Person auffallend selten ist, weil vom verstorbenen König in den vorliegenden Fassungen in der 3. Person gesprochen wird ${ }^{27}$, machen die Vorkommen des Pseudopartizips in der 1. Person in den Autobiographien des Alten Reiches mehr als die Hälfte aus.

${ }^{27}$ I. Pierre, La gravure des textes dans la pyramide de Pépi $\mathrm{I}^{\text {er }}$. Les différentes étapes, in: C. Berger et al. $(\mathrm{Hg}$.), Hommages à Jean Leclant (BdÉ 106), Le Caire 1994, Bd. 1, 306 f.; B. Mathieu, Modifications de texte dans la pyramide d'Ounas, in: BIFAO 96, 1996, $291 \mathrm{f}$. 
In etlichen Fällen kommen telische Verben in aktiver Diathese vor, vorwiegend in der 1. Person (2. Person in deontischem Modus in Bsp. 31). Die Beleglage deutet darauf, dass es Subjekte sind, die in der Hierarchie der salience auf sehr hoher Stufe stehen, die mit der aktiven Diathese in Verbindung stehen. Vgl. oben Abschnitt 1.1.

In wenigen Beispielen ist das Verb auf den ersten Blick intransitiv, also ohne direktes Objekt, verwendet. Das in Bsp. 23 verwendete Verb $d b h m^{r}$, ,jemanden bitten“, kann aber nicht als Beleg gelten, da die Grundbedeutung intransitives „bedürftig sein“ ist. Die Verwendung mit $m-{ }^{-}$ist idiomatisch, wie der Vergleich mit Bsp. 24, mit $s \underline{d m}=f$-Form, zeigt.

23. [d]bh.k(wj) $m-{ }^{\ulcorner} h m n n b=j j n(j) . t(w) n(=j) j n r h d$ $k r s,,[\ldots]$ da bat ich bei der Majestät meines Herrn darum, dass mir weißer Stein (für) den Sarg gebracht werde $e^{c^{28}}$.

24. $h r d b h(=j) m-^{c} n b=j j n(j) . t(w) j n r \quad h d \underline{d} k r s w n j t=j$ $p n$, „Dann bat ich bei meinem Herrn darum, dass weißer Stein (für) den Sarg gebracht werde für diesen meinen Vater ${ }^{c{ }^{29}}$.

Im folgenden Fall begegnet das Verb $r d j$, „geben“, im Sinne von „veranlassen“, dem in der Tiefenstruktur nicht ein eingebetteter Objektsatz folgt, sondern ein eigenständiger Satz: „Ich gebe (Anweisung): er soll ..." resp. eine direkte (nicht indirekte) Rede. Es handelt sich um eine Kausativierung, die analytisch realisiert ist. Das Verb $r d j$ in seiner kausativen Funktion ist syntaktisch gesehen aktiv-intransitiv, semantisch hingegen transitiv.

25. $r$ dj.kt(wj) $f(j) . t(w)=f$ jn $\underline{t s . w t}$ n.t $\mathrm{pr} n \underline{\mathrm{d}} . t(=j)$, ,Ich veranlasste, dass er von den Truppen meiner Totenstiftung transportiert wurde ${ }^{6 s^{30}}$.

In den übrigen Fällen mit aktiver Bedeutung kommt ein direktes Objekt vor.

26. $k r s . k(w j) s p n m j s=f m h(t j) N h b$, ,Ich bestattete diesen Mann in seinem Grab nördlich von el$\mathrm{Kab}^{6{ }^{31}}$.

27. w3h.k(wj) jnw $n b$, ,IIch fuhr nach Memphis mit den Gaben jener Länder, die dieser Hatia gebracht hatte.) Ich legte alle Gaben nieder ${ }^{43^{32}}$.

\footnotetext{
${ }^{28}$ Urk. I 99, $10 \mathrm{f}$.

${ }^{29}$ Urk. I 65, $12 \mathrm{f}$.

${ }^{30}$ Urk. I, 136, 17.

${ }^{31}$ Urk. I 140, 8.
}

28. $s[n] h . k(w j)$ ts.t [tn], „Ich verpflegte diese Trup$\mathrm{pe}^{\mathrm{sis}}$.

29. $\operatorname{sh} 3(0) . k(w) n=f h t p p n n h r w 17$ wh3 $(. w) m h w . t-n b$ [...] $\xi . k(w j) n=f$ wsh.t $m$ šndn.t $m h .60 \mathrm{~m} 3 w=s \mathrm{mh}$ $30 m w s h=s$, ,[Seine Majestät sandte mich nach Hatnub, um einen großen Opfertisch aus Hatnub-Alabaster herbeizubringen.] Ich führte ihm (dem König) diesen Opfertisch in 17 Tagen he$\mathrm{rab}$, nachdem er in Hatnub gebrochen worden war. [...] Ich haute für ihn (den Opfertisch) eine Barke aus Akazienholz von 60 Ellen Länge und 30 Ellen Breite ${ }^{\text {c.6. }}$.

30. $j r(j) . k(w j) n=f j m j-r 3 m^{\top} w^{2}$, ,Ich machte für ihn den Vorsteher von Oberägypten“ $\rightarrow$ „Ich übte für ihn (das Amt eines) Vorstehers von Oberägypten aus / Ich bewährte mich ihm gegenüber als Vorsteher von Oberägypten “35.

31. wnh.t(j) $\underline{d . t} t=k$, , Mögest du mächtig sein in deinem Leib.] Mögest du deinen Leib bekleiden" $\rightarrow$ „Mögest du dich bekleiden ${ }^{\text {‘6 }}$.

Betrachtet man die als direktes Objekt involvierten Größen, so zeigt sich folgendes: Sie sind anaphorisch-deiktisch (Bsp. 26, 28f.), anaphorisch mit Quantifikator (Bsp. 27) und semantisch reflexiv (Bsp. 30f.). Sie sind somit sämtlich ko(n)textuell gegebene Größen.

Die semantische Marginalisierung des Agens muss in Sätzen wie Bsp. 26-29 mit direktem Objekt und mit einem Verb, das ein accomplishment ausdrückt, zur Kausativierung führen ${ }^{37}$. Das direkte Objekt bezeichnet das Patiens der kausativierten Handlung (nicht das Patiens der Kausativierung), weswegen das Verb passiv zu denken ist.

26. „Ich ließ diesen Mann bestattet werden",

27. "Ich ließ alle Gaben niedergelegt werden",

28. „Ich ließ diese Truppe verpflegt werden“,

${ }_{33}^{32}$ Urk. I, 139, 5.
Anthes, Felsinschriften (wie Anm. 21), Taf. 10, Gr. 4, Kol. 5.

${ }^{34}$ Urk. I, 108, 1; Biographie des Uni: T. Hofmann, Die Autobiographie des Uni von Abydos, in: LingAeg $10,2002,228$, Kol. 44f. Vgl. Kol. 22: $m 3^{r} . k(w j) s n m$ $[\ldots]$, ,Ich führte sie nach $[\ldots .$. ]".

Urk. I, 106, 4; Hofmann, Autobiographie des Uni (wie Anm. 34), 228, Kol. 36. - Etwa entsprechend der französichen, in der 3. Person möglichen Wendung se faire ..., ,... werden".

${ }_{36}$ Pyr. 1300c $(537, \mathrm{P})$

${ }^{37}$ Vermutet von W. Schenkel, Das altägyptische Pseudopartizip und das indogermanische Medium/ Perfekt, in: Or 40, 1971, 307. 
29. „Ich ließ diesen Opfertisch für ihn hinabgebracht werden [...] ich ließ für ihn eine Barke gehauen werden".

Als deutsche Übersetzung mit Infinitiv eignet sich „Ich ließ diesen Mann bestatten“" etc. wohl dennoch besser. Es handelt sich um eine auf das Patiens bezogene Kausativierung ohne Möglichkeit einer Valenzerhöhung, d.h. es wäre mit dieser Konstruktion nicht möglich zu sagen „Ich ließ A diesen Mann bestatten" (s. o.) und auch nicht „Ich ließ diesen Mann von A bestattet werden". Intransitive Verben müssen in dieser Konstruktion durch das kausativierende $s$-Präfix transitiviert werden (Bsp. 28f.).

Das kausative Pseudopartizip (Bsp. 26-29) hat für die kausativierte Handlung nur ein patient-slot. Die Kausativierung durch $s$-Präfix dagegen hat für die kausativierte Handlung nur ein subject-, aber nicht gleichzeitig ein object-slot (sh3j, „veranlassen, dass $\mathrm{S}$ hinabgeht", $s m s j$, „veranlassen, dass S gebiert", nicht: „veranlassen, dass S O gebiert"), so dass ein $s$-Kausativ keinen ,doppelten Akkusativ" hat ${ }^{38}$. Es handelt sich bei dieser Konstruktion um direkte Kausation ${ }^{39}$. Seltener scheinen Fälle zu sein, wo nur ein patient-slot vorhanden ist (smsj, „veranlassen, dass $\mathrm{P}$ erzeugt ist $\left.^{\circ}\right)^{40}$. Diese Konstruktion dient primär der Transitivierung eines intransitiven Verbs, d. h. der Valenzerhöhung (Bsp. 28f.), oder der Verdeutlichung der faktitiven Transitivität ${ }^{41}$ ambiva-

${ }^{38}$ Z. B. Pap. Westcar 11,4: sms $(j)=s n$ Rwd-dd.t $m p^{3}$ hrd.w 3, "nachdem sie Ruddjedet von den 3 Kindern entbunden hatten".

${ }^{9}$ Vgl. W. Schenkel, s-Kausativa, $t$-Kausativa und „innere" Kausativa. Die s-Kausativa der Verben I. $s$ in den Sargtexten, in: SAK 27, 1999, $318 \mathrm{f}$. Zu direkter und indirekter Kausativierung siehe z. B. W. Frawley, Linguistic Semantics, Hillsdale 1992, 164-170.

${ }^{40}$ Z. B. DZA 29243890 (http://aaew.bbaw.de/dza/ index.html): $r s m s(j) p 3 w t j . w m p 3 w . t=k$ tpj.t, „um die Urzeitlichen entstehen zu lassen aus deiner Urzeit“; DZA $29243840 n b h w s m s(j) d \beta$, „der Herr der Nahrung, der Speise entstehen lässt". Möglicherweise handelt es sich um eine nachmittelägyptische Entwicklung, überdies scheint die Produktivität der $s$-Kausative im Spätmittelägyptischen wiederbelebt worden zu sein, vgl. K J ansen-Winkeln, Spätmittelägyptische Grammatik der Texte der 3. Zwischenzeit (AAT 34), Wiesbaden 1996, $55, \int 88$.

Vgl. Edel, Altägyptische Grammatik (wie Anm. 10), $194 \mathrm{f}$., $₫ 440$; Schenkel, $s$-Kausativa (wie Anm. 39), 319 lenter Verben (Bsp. 32f.). Wenn $w^{\text {}} b$, ,(sich) waschen/reinigen", agentivisch verwendet ist (vgl. Abschnitt 1.1 mit Bsp. 8f.) unter Angabe des direkten Objektes (Bsp. 9 und 33) und allenfalls unter expliziter Angabe des Instrumentes (Bsp. 33), wird das Pseudopartizip nicht verwendet - auch dann nicht, wenn die Handlung faktitiv und perfektiv ist. In diesem Fall kann die Kausativbildung $s w^{`} b$, „waschen/reinigen", der Verdeutlichung dienen (Bsp. 32f.).

32. $w^{c} b \zeta m s w ~ H r$ N.N. $p n s^{e} b=s n\left(\right.$ Var. $\left.w^{\tau} b=s n\right)$ N.N. $p n s \check{s} w(j)=s n$ N.N. pn, „Die Anhänger des Horus waschen diesen N.N., sie versetzen diesen N.N. in reinen Zustand und trocknen diesen N.N. $a b^{6{ }^{42}}$.

33. $s w^{c} b \underline{t} w \mathrm{Hr} m k b h w$, „Horus hat dich mit kühlem Wasser gereinigt ${ }^{\circ 4}$.

Zu Bsp. 26-31 einerseits und Bsp. 32f. andererseits seien folgende Sätze verglichen:

25. $r \underline{d j} . k(w j) \beta(j) . t(w)=f j n \underline{t s} . w t$ n.t $p r n \underline{d} . t(=j)$, „Ich veranlasste, dass er von den Truppen meiner Totenstiftung transportiert wurde".

34. $r \underline{d}(j) h m=f$ wrh.t(t $w=j) m$ ' $n d$, „Seine Majestät veranlasste, dass ich mit 'nd-Öl gesalbt wur$\mathrm{de}^{\mathrm{es}}$.

Bsp. 25 und 34 verwenden zum Ausdruck der Kausativierung die analytische Konstruktion mit übergeordnetem $r \underline{d j}$. In beiden Sätzen ist das Patiens genannt (als Subjekt des subjunktivischen $t w$-Passivs), in Bsp. 25 auch das semantisch extra markierte Agens. Die analytische Konstruktion mit $r d j$ hat, ob passiv, wie hier, oder aktiv formuliert und entsprechend der $\mathrm{Au}$ tonomie des kausativierten Verbs, sowohl den agent- wie auch den patient-slot $t^{45}$. Bsp. 25 verwendet diese Konstruktion und nicht das kausative Pseudopartizip, weil sowohl Agens wie auch Patiens der kausativierten Handlung angegeben werden, und auch nicht ein $s$-Kausativ, weil es um eine indirekte Kausativierung geht. Bsp. 34 verwendet die Konstruktion mit $r \underline{d j}$ und nicht

${ }^{42}$ Pyr. 921a-b (Spr. 471, P, Var. N).

${ }^{43}$ Pyr. 841 b (Spr. 452, P).

${ }^{44}$ Urk. I, 60, 4.

${ }^{45}$ Z. B. Pyr. 584 a (Spr. 357, T): $r \underline{d}(j) . n$ Hr j.nd $\underline{t} w$ 3s.t $h n^{r} N b . t-h w . t$, „Horus hat veranlasst, dass Isis und Nephthys dich schützen“; Stele, Kairo, CG 20538, II, Z.9: $d j=j r h=\underline{t} n s h r n n h h$, „Ich lasse euch einen Ratschlag für die Zukunft wissen“". 
das kausative Pseudopartizip, weil dies in der 3. Person nicht möglich wäre. (Zur salience vgl. Abschnitt 1.1 und 4.)

Während bei agentivischen Verben mit einem direkten Objekt wie in Bsp. 26-29 also die semantische Marginalisierung des Agens zur Kausativierung führt, verwenden Bsp. 30f. activity-resp. state-Verben mit semantisch reflexivem Objekt. Sie sind somit nur syntaktisch gesehen aktiv-transitive Sätze.

Zum Vergleich seien folgende Personennamen aus dem Alten Reich aufgeführt, aus denen ersichtlich ist, dass ein Verb, dessen Subjekt mit dem Agens koreferentiell ist, nicht mit dem Pseudopartizip ausgedrückt werden kann, wenn es aktive Bedeutung hat (Bsp. 35). Atelische Verben hingegen, für die eine Agensrolle nicht vorgesehen ist, stehen im Pseudopartizip (Bsp. 36).

35. $k 3(=j) \quad c h 3=f / m r(j)=f / h w j=f$, ,Mein Ka sei kampfbereit / sei willensstark / sei schlagkräftig ${ }^{\star c+6}$.

36. $k 3(=j) j j(. w) /{ }^{2} p r(. w) / \complement_{n h(. w) / w} b(. w) / w h m(. w) /$ $w \underline{d} 3(. w) / m n j(. w) / n f r(. w) / r s(. w) / s \underline{d} 3(. w) / \underline{t} n j(. w)$ / $t s j(. w)$, „Mein $\mathrm{Ka}$ ist gekommen / sei gerüstet / lebe / sei rein / wiederhole sich / sei heil / bleibe / sei vollkommen / sei wach / sei in Bewegung / sei aufgerichtet / sei erhoben “677.

$\mathrm{Zu}$ Bsp. 26 ist ein semantisch fast identischer Satz zu zitieren, der aber die $s \underline{d} m . n=f$-Form verwendet:

26. $k s s . k(w j) s$ pn $m j s=f m h(t j) N h b$, „Ich ließ diesen Mann in seinem Grab nördlich von el-Kab bestatten".

37. $j w \quad k r s . n(=j) j t(=j)$ pn $m$ js=f $n$ hr.t-ntrr, „So bestattete ich diesen meinen Vater in seinem Grab der Nekropole ${ }^{c 48}$.

In seiner Autobiographie berichtet Sabni ausführlich vom Tod seines Vaters im Ausland und von den Bestattungsvorbereitungen. Satz 37 steht zwischen der Wiedergabe des Wortlauts eines Dekrets zugunsten des Begräbnisses und der Schilderung der aufwendigen Vorbereitungen Sabnis; die Bestattung ist an diesem Punkt

\footnotetext{
${ }^{46}$ PN I, 338, 26; 240, 3; 15.

${ }^{47}$ PN I, 338, 18f.; 24f.; 339, 2f.; 6f.; 340, 2; 10; 13; 341,$1 ; 3 \mathrm{f}$.

${ }^{48}$ Urk. I, 139, 1.
}

des Textes noch nicht vollzogen. Die $s \underline{d m} . n=f$ Form ist ein präsentisches Perfekt; sie dient nicht dazu, den Abschluss der Handlung festzustellen. In Bsp. 26 dagegen heißt es, Sabni habe für jemandes Begräbnis gesorgt. Nach dem zitierten Satz wird von einer Auszeichnung Sabnis berichtet. Damit ist die erzählte Episode zu Ende. Der Aspekt des Pseudopartizips ist perfektisch ${ }^{49}$.

Nun ist noch das Verb $r h$ zu betrachten, dessen Bedeutung üblicherweise mit „etw. kennen/erfahren" angegeben wird - eine Bedeutung, die dank der ihr inhärenten epistemischen Modalität auch in der allgemeinen Linguistik semasiologisches Interesse erfährt ${ }^{50}$. Die Bedeutung des Pseudopartizips müsste dann passiv sein: „er ist erfahren worden/man kennt ihn“. Legt man hingegen als Bedeutung von $r h$,kun$\mathrm{dig} /$ wissend werden/sein" zugrunde, so kommt man auf die in diesem Fall zutreffende Bedeutung des Pseudopartizips „wissend/kundig geworden sein“, also rh.w, „er weiß/kennt“. Die Frage ist somit häufig, von welcher Übersetzung man (zufällig) ausgeht. Und damit verbunden: Welches ist die anzunehmende Grundbedeutung eines Verbs, wenn die Wörterbücher von einem Lexem transitive, intransitive (wie auch reflexive) Bedeutung aufführen (z. B. $w^{c} b$, ,rein sein/sich-reinigen/reinigen")? Im Vergleich zu den übrigen Beispielen mit direktem Objekt (Bsp. 26-31) ist $r h$ in seiner Grundbedeutung kein agentivisches Verb. Die Kausativität des Pseudopartizips mit direktem Objekt ist hier

${ }^{49}$ Während Edel, Altägyptische Grammatik (wie Anm. 10), 285, 5 590, den zweiten Satz als gleichbedeutend mit dem vorangehenden Bsp. bezeichnet, deuten M. Malaise/J. Winand, Grammaire raisonnée de l'égyptien classique (AegLeod 6), Liège 1999, 447, \$ 722 , die beiden Sätze just andersherum: „La première forme, grâce à l'auxiliaire $i w$, marque l'intérêt que prend le sujet à l'action, puisqu'il s'agit de son propre père; la seconde forme est purement narrative". Das eigene Interesse müsste bei der Deutung des Pseudopartizips als Medium im Gegenteil bei dem zweiten Satz vorliegen. Das ist auch nicht auszuschließen; Sabni wird nach der Ausführung belohnt.

${ }^{\text {so }}$ Z. B. Z. Vendler, Verbs and Times, in: A. Schopf (Hg.), Der englische Aspekt, Darmstadt, 1974, 217-234. Vgl. auch unten zu Bsp. 66f.; Frawley, Linguistic Semantics (wie Anm. 39), 412-415. 
innerlich reflexiv (,sich selbst wissend werden lassen"). Als Verb der inneren Wahrnehmung verlangt die Bedeutung ein nichtkoreferentielles Patiens (,sich selbst etwas wissend werden lassen"), das an der Oberfläche nicht durch eine Präposition bezeichnet wird (vgl. „sich informiert haben $\left.\ddot{u} b e r^{r}\right)$, sondern wie ein direktes Objekt eines Verbs in aktiver Verwendung erscheint (,sich eine Information zukommen lassen haben/etwas kennen").

38. $d d m d w j . r h . k(w j) r m=k$, ,Zu rezitieren: Ich habe veranlasst, dass ich deinen Namen wissend werde“ $\rightarrow$ „Ich habe mich deinen Namen wissend werden lassen" $\rightarrow$ „Ich weiß/kenne deinen Namen “ ${ }^{\text {s1 }}$.

Diese reflexive Kausativierung wird auch auBerhalb der 1. Person gebraucht. In den Pyramidentexten kommt das Verb in der $s d m . n=f$-Form nicht vor, in der $s \underline{d m}=f$-Form nur negiert (vgl. auch Abschnitt 1.4). Das so verwendete Pseudopartizip ist prädestiniert für das semantische Feld von nichtspezifiziertem „Wissen“, d. h. auf eine Information, die nicht eingeschränkt ist auf direktes oder aus erster Hand erworbenes Wissen - eine Unterscheidung, die in gewissen Sprachen gemacht wird -, da das Agens im Pseudopartizip eine untergeordnete Rolle spielt: "Ich weiss, aber die Information kommt von irgendwoher; ich habe mich selbst informiert, aber nicht in dem Sinne, dass ich die Quelle der Information bin; ich bin einfach informiert“.

\subsection{Die Rolle des Benefaktivs}

Das altägyptische Pseudopartizip hat, wie gezeigt, grundsätzlich die folgenden vier syntaktisch-semantischen Verwendungsmöglichkeiten (mit Angabe typischer Beispiele):

a. intransitive Verwendung, nonagentivisches Verb, d. h. Subjekt nicht in der semantischen Rolle des Agens (Bsp. 4: [...] jw $f w^{c} b(. w),,[\ldots]$ er ist rein"),

b. transitiv-passive Verwendung, agentivisches Verb, Subjekt in der semantischen Rolle des Patiens, Agens nicht genannt, d. h. semantisch

\footnotetext{
${ }^{51}$ Pyr. 1434a (Spr. 569, P).
}

vollständig marginalisiert (Bsp. 6: [...] nd.tj [...], „[...] du sollst geschützt sein [...]"),

c. transitiv-passive Verwendung, agentivisches Verb, Subjekt in der semantischen Rolle des Patiens, Agens genannt und syntaktischmorphologisch marginalisiert durch Extramarkierung mit $j n$ (Bsp. 15: $h s(j) . k(w j) h r=s j n$ $n b=j$, „Ich wurde ausgezeichnet deswegen von meinem Herrn"),

d. transitiv-kausative Verwendung, agentivisches Verb, Subjekt gleich semantisch durch Kausativierung und hohe salience marginalisiertes Agens (1. Person), direktes Objekt in der Rolle des Patiens mit hoher salience (Bsp. 26: krs.k(wj) s pn [...], „Ich ließ diesen Mann bestattet werden [...]"); dazu als Subkategorie die reflexivkausative Verwendung bei $r h$, „wissen/kennen“".

Bei der eingangs erwähnten medialen Diathese geschieht die Handlung im Interesse der von der Handlung betroffenen Größe, d.h. das Benefaktiv (englisch benefactive oder beneficient, Nutznießer) ist mit dem Subjekt koreferentiell. Das auf das Subjekt bezogene Benefaktiv wird bei medialen Formen somit nicht extra, außerhalb der Verbalform realisiert ${ }^{52}$. Dies ist beim Pseudopartizip der Fall. Bsp. 6 lautet nicht *[...] $n$ d.tj $n=k[\ldots],[\ldots]$ du sollst geschützt sein $z u$ deinen Gunsten $[\ldots]^{\text {‘. }}$.

Wenn das Pseudopartizip medial ist, dürfte ein Benefaktiv, das nicht mit dem Subjekt koreferentiell ist, nicht vorkommen. Das Pseudopartizip hat sozusagen nur Interesse an sich selbst und das Agens ist ihm egal; dieses wird semantisch oder syntaktisch marginalisiert. Dagegen ist zu halten: ,[...] benefactives typically require an agent elsewhere in the predication ${ }^{66^{53}}$. Außer im Fall d liegen sehr häufig stativische Verben vor oder aber der Verbalinhalt erfährt durch die Passivierung bei perfektivem Aspekt gleichzeitig auch Stativierung (,er ist gesättigt worden“" $\rightarrow$ „er ist gesättigt/satt"). „Benefactives co-occur with agents and thus are likely to be found in nonstative predicates, especially nonstative pre-

52 S. Kemmer, The Middle Voice, Amsterdam/Philadelphia 1993, 37-39; 74-81. Vgl. auch Jenni, Die pronominalen Erweiterungen (wie Anm. 17), 117.

${ }_{55}$ Frawley, Linguistic Semantics (wie Anm. 39), 216, mit Bezug auf C. Fillmore, The Case for Case, in: E. Bach/R. T. Harms (Hg.), Universals in Linguistic Theory, New York 1968, 1-88. 
dicates of result ${ }^{654}$. Somit ist zu erwarten - und zeigt sich -, dass bei Nennung eines Benefaktivs (ausgedrückt durch die Präposition $n$ ), das nicht mit dem Agens koreferentiell ist, die $s \underline{d m}=f$ oder die $s \underline{d m} . n=f$-Form eintritt.

19. $j r(j . w) n=f n m . t \check{s}$, ,Möge für ihn die (Zeremonie der) Überfahrt über den See ausgeführt werden".

39. jnk.n $n=k$ Nb.t-hw.t ${ }^{\ulcorner} . w t=k n b . t[\ldots] s w d 3 . n<=s>$ $\boldsymbol{n}=\boldsymbol{k} s \boldsymbol{s}$, ,Nephthys hat für $\mathrm{dich} / \mathrm{zu}$ deinen Gunsten alle deine Glieder zusammengefügt [...] und hat sie $\operatorname{dir} / z u$ deinen Gunsten geheilt ${ }^{455}$.

40. $\underline{d} d \boldsymbol{d} d w$ snht $n=k$ p.t $j 3 h w w,, Z \mathrm{u}$ rezitieren: Möge der Himmel für dich/zu deinen Gunsten den Lichtglanz verstärken ${ }^{\text {c56 }}$.

Im Falle des deontischen Modus (Bsp. 40) ist der Imperativ, zumindest ursprünglich, kein Ersatz für den genannten Sachverhalt, da dort ein $j n=k \rightarrow n=k$ plus direktes Objekt eigentlich das Agens verstärkt ${ }^{57}$.

Natürlich dient nicht jedes $n+$ Nominalphrase zum Ausdruck eines Benefaktivs. Bei Verben der Bewegung geben sie das Ziel an, wenn dieses eine Person (und nicht ein Ort) ist (Bsp. 41). Allerdings findet das Pseudopartizip typischerweise da Verwendung, wo das Ziel mit dem Subjekt koreferentiell ist (Bsp. 42: „zu deiner Seele“ $\rightarrow$ „zu dir").

41. $j j(j) n=k$ N.N. $p w m j w n w$, ,Dieser N.N. möge zu dir (Osinis?) kommen in Heliopolis ${ }^{458}$.

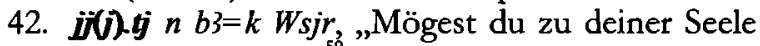
kommen, Osiris! ‘50.

Bei dreiwertigen Verben wie $j n j$, ,jemandem etwas bringen“, oder $r d j$, ,jemandem etwas geben", besetzt das mit $n$ eingeleitete indirekte Objekt üblicherweise die vorgesehene dritte Rolle des Empfängers (recipient), nicht die (zusätzliche) Rolle eines Benefaktivs. Es finden sich von dem Verb $r d j$ nur ganz wenige Vorkommen im Pseudopartizip (Bsp. 44) und nur, wenn ein 216.

${ }^{54}$ Frawley, Linguistic Semantics (wie Anm. 39), ${ }^{55}$ Pyr. 616a-c (Spr. 364, T).

${ }^{56}$ Pyr. 1231 a (Spr. 523, M).

57 Jenni, Die pronominalen Erweiterungen (wie Anm. 17).

${ }^{58}$ Pyr. 2097c (Spr. 690, N).

${ }^{59}$ Pyr. 215b (Spr. 223, W).
Agens nicht explizit genannt ist. Üblicherweise sind die Formen als Passive der Suffixkonjugation zu erkennen oder zu deuten.

43. jnj.n $n=k s n H r s s^{r} . w$, „Horus hat sie dir gebracht, indem sie abgeschnitten waren ${ }^{\text {600 }}$.

44. $r d(j) . t(j) n m w t=k$ Nw.t $m r n=s \quad n k r s w . t$, ,so dass du gegeben bist deiner Mutter Nut in ihrem Namen,Bestattung " ${ }^{61}$.

45. $r \underline{d y} \quad n=f \quad 3 h=f j m \quad r d y \quad n=f \quad s h m=f j m$, ,Seine Geistkraft ist ihm gegeben worden dabei, seine Macht ist ihm gegeben worden dabei ${ }^{*{ }^{62}}$.

In den folgenden beiden Sätzen mit dem Verb $j n j$ hingegen bezeichnet $n$ nicht den Empfänger im gewöhnlichen Sinn, sondern das Benefaktiv, den Nutznießer, denn es handelt sich um einen Verstorbenen, für den die Vorbereitungen zu seinem Begräbnis ausgeführt werden. Obwohl $j n j+n$ in beiden Fällen im subjunktivischen $t w$-Passiv steht, ist übergeordnet in Bsp. 23, wo ein Sarg für die bittende Person selbst erbeten wird, das Benefaktiv also mit dem Agens koreferentiell ist, das Pseudopartizip verwendet. Anders in Bsp. 24 mit der übergeordneten $s \underline{d} m=f$-Form, wo der Autor einen Sarg für eine Person erbittet, die nicht mit ihr koreferentiell ist.

23. [d]bh. $k(w j) m-\ulcorner h m n n b=j j n(j) . t(w) n(=j) j n r h \underline{d}$ $k r s$, „[...] da bat ich bei der Majestät meines Herrn darum, dass mir weißer Stein (für) den Sarg gebracht werde“.

24. $h r d b h(=j) m-r n b=j j n(j) . t(w)$ jnr had krsw $n j t=j$ $p n$, „Dann bat ich bei meinem Herrn darum, dass weißer Stein (für) den Sarg gebracht werde für diesen meinen Vater".

In den folgenden beiden Sätzen, ebenfalls mit Pseudopartizip in aktiver Bedeutung, bezeichnet das zweimal vorkommende anaphorische und pronominale $n=f$ nicht das Benefaktiv. Der Vorgesetzte ist in Bsp. 29 der Auftraggeber, an den der bestellte Gegenstand geliefert wird - insofern gleicht das Verb $s h 3 j$ den Verben $j n j$ und $r \underline{d j}$. In Bsp. 30 ist die Handlung sozusagen die Ausführung der Ernennung durch den Vorgesetzten und nonagentivisch, da das direkte Objekt nicht das Produkt der Handlung, sondern,

\footnotetext{
${ }^{60}$ Pyr. 653b (Spr. 372, T)

${ }^{61}$ Pyr. 616d (Spr. 364, T)

${ }^{62}$ Pyr. 857c (Spr. 475, N).
} 
wie bereits oben festgestellt, semantisch reflexiv mit dem Subjekt ist.

29. $\operatorname{sh} 3(j) . k(w j) n=f h t p$ pn $n h r w 17$ wh3(.w) $m$ hw.t-nb [...] $s$. $k(w j) n=f$ wsh.t $m$ šndn.t $m h .60 m 3 w=s m h$ $30 m$ ws $h=s$, ,[Seine Majestät sandte mich nach Hatnub, um einen großen Opfertisch aus Hatnub-Alabaster herbeizubringen.] Ich ließ (dem König) diesen Opfertisch in 17 Tagen zu ihm herabführen, nachdem er in Hatnub gebrochen worden war. [...] Ich ließ für ihn (den Opfertisch) eine Barke aus Akazienholz hauen von 60 Ellen Länge und 30 Ellen Breite".

30. $j r(j) . k(w j) n=f j m j-r 3 m^{c} w$, „Ich machte für ihn den Vorsteher von Oberägypten“ $\rightarrow$ „Ich übte für ihn (das Amt eines) Vorstehers von Oberägypten aus".

Sofern $n f r$ im folgenden Beispiel als Pseudopartizip $z u$ interpretieren ist, böte es einen $\mathrm{Ge}$ genbeweis, wo tatsächlich ein Benefaktiv zusammen mit einem Pseudopartizip vorkommt: ein Pseudopartizip der 3. Person, deren Referentialität gleich null ist.

46. htp ntr.w $n b(. w)$ hr (j)h.t $n b(. t)$ j.d dd.t N.N. pn $n f r(. w) n=f j m$ d.t.t $n$ d.t.t, ,Alle Götter sind zufrieden mit allem, was dieser N.N. gesagt hat. Es steht gut dadurch für ihn für immer und immer ${ }^{463}$.

In den Bsp. 8 und 47f. geht es um die Feststellung kultischer Reinheit am nonagentivischen Ende der Skala (faktitiv und perfektisch). Was nach der Präposition $n$ folgt, ist eine göttliche Größe. Nonagentivisch kann man sich gereinigt haben/rein sein "für" $(n)$ nicht im Sinne von „zu Gunsten von“, sondern im Sinne von „gegenüber von“ einer göttlichen Größe (Bsp. 47). Dass die Präposition $n$ so gemeint ist, zeigt die Variante mit der Präposition $r$ (Bsp. 8) im Gegensatz zu Bsp. 48 mit $n$ und $s d m=f$-Form (negiert).

47. $w^{\ulcorner} b(. w) n=\underline{t} \operatorname{snd}(. w) n=\underline{t},,[\ldots]$ indem er (König) rein ist dir (Krone) gegenüber und indem er voll Furcht ist dir gegenüber ${ }^{16^{64}}$.

\footnotetext{
${ }^{63}$ Pyr. 1645c (Spr. 599, N). Bei J. P. Allen, The Inflection of the Verb in the Pyramid Texts, Malibu 1984, 617, $\int 773$, unter den Pseudopartizipien eingeordnet. Faulkner, The Ancient Egyptian Pyramid Texts (wie Anm. 16), 246, übersetzt: „[...] by means of which it goes well with him for ever and ever".

${ }^{64}$ Pyr. 194d (Spr. 220, W).
}

8. wrb.w $r=j$ nht $j b=s n r=s$, ,[...] indem sie rein sind mir gegenüber ${ }^{65}$ und indem ihr Herz stark ist dabei"

48. $n(j) w^{c} b=s n n=j w^{c} b=s n n 3 h j k r,,[\ldots]$ indem sie nicht rein sind für mich, wie sie rein sein sollten für einen trefflichen Verklärten ${ }^{666}$.

\subsection{Zur Negierung des Pseudopartizips}

Das Pseudopartizip kommt äußerst selten mit Negation vor. Das ist verständlich aufgrund des perfektiv-perfektischen Aspekts: Nicht den Verlauf, sondern (nur) den erreichten Zustand zu negieren, wäre wenig sinnvoll, weswegen im Falle einer Negierung die $s \underline{d} m=f$ - oder die $s \underline{d m . n}=f$-Form verwendet wird.

Denkbar wäre in einem Satz mit Pseudopartizip aber wohl die Negation einer Adverbialphrase durch die konträre Negation $n j$... js:

49. j.hmsj.tj hr ns.t jt=k Gb m-hnt jtr.t, ,Du sollst auf dem Thron deines Vaters Geb sitzen inmitten der Nische ${ }^{66^{67}}$.

50. ${ }^{*} n(j) j . h m s j . t j ~ j s ~ h r r n s . t j t=k G b m-h n t ~ j t r . t j . h m s j . t j$ $h r$ ns.t $j t=k G b$ [AP], „Du sollst nicht auf dem Thron deines Vaters Geb inmitten der Nische sitzen, sondern du sollst auf dem Thron deines Vaters Geb [AP] sitzen“.

Im Mittelägyptischen kommen - selten - negierte pseudoverbale Konstruktionen mit Pseudopartizip vor. Doch ist dann die Aussage konträr, die Verbalform an sich bleibt positiv: „es ist nicht der Fall: es ist nützlich für dich“ (Bsp. 51); „es gibt keinen: er ist existent" (Bsp. 52) ${ }^{68}$.

51. $m \operatorname{skr(.w)} n n$ st $3 h(. w) n=k$, ,[Hüte dich vor ungerechter Bestrafung,] schlag nicht zu, (wenn) es nicht nützlich für dich ist! ${ }^{\text {(69 }}$.

52. $n n s w w n(. w)$, , [Zu wem soll ich heute sprechen? Es gibt keinen Zufriedenen, und jener, mit dem man ging -] er ist nicht existent ${ }^{6{ }^{70}}$.

${ }^{65}$ Edel (wie Anm. 10) übersetzt: ,indem sie rein sind für mich", doch hat die Präposition $r$ nicht die Bedeutung ,für"; vgl. H. Jenni, ,Sagen zu“ im Ägyptischen, Lingua Aegyptia, Bd. 10, 2002, $246 f$.

${ }^{66}$ Urk. I, 202, 5.

${ }^{67}$ Pyr. 1192b (Sp. 673, N).

${ }^{68}$ Hinweis von Antonio Loprieno, dem für eine begriffsklärende Diskussion dieser Arbeit herzlich gedankt sei.

${ }^{69}$ Merikare E 48.

${ }^{70}$ Lebensmüder $126 f$. 
Die seltenen kontradiktorisch negierten Pseudopartizipien finden sich in den Pyramidentexten. Am ersten Zitat zeigt sich überdies, dass die Stellung eines nominalen Subjekts im Altägyptischen aus prosodischen Gründen variabel ist.

53. $d d m d w s d 3(j) N . N . p n h n^{c}=k H r$, ,Zu rezitieren: Nimm den N.N. da mit dir, Horus", $d 3(j) s w$ Dhwtj $m$ tp ${ }^{\prime} n d=k S k r$ js hnt $(j) m 3{ }^{c} . t$, „fahre ihn hinüber, Thot, auf deiner Schwinge als Sokar, dem Ersten der Maat(-Barke)“, $n(j) H r s d r(. w) h 3$ $m r n(j) j w y \underline{w} h w t j$, ,Horus schläft nicht hinter dem Kanal, Thot ist nicht ohne Boot", $n(j) \mathrm{hm}$ jwj.w N.N. pn N.N. pw hr jr.t Hr, ,Auch der N.N. da ist nicht ohne Boot; der N.N. da trägt das Horusauge $e^{66^{71}}$.

54. $n(j) s 3$ (J)tm jwy, „Der Sohn Atums ist nicht ohne Boot ${ }^{4 c^{72}}$.

Die beiden mit der kontradiktorischen Negation $n(j)$ verwendeten Verben sind in ihrer Grundbedeutung negativen Inhalts. Bei $j w j$, „bootlos sein", ist dies unmittelbar einsichtig. Das Verb $s \underline{d} r$, häufigste Bedeutung „liegen/die Nacht verbringen/schlafen“, ist als Kausativ des Verbs $\underline{d} r \mathrm{zu}$ verstehen, dessen Bedeutung „(den Fuß/Tritt) anhalten" (Wb V, 595, 5-9) ist. Die Bedeutung von $s \underline{d r}$ ist also kausativ-reflexiv: „sich inaktivieren“. Das wird durch folgendes Bsp. verdeutlicht:

55. $d d m d w h r g s=k s d r . t j h t(j) \cdot t j h t(j) . t j m j w m j w,,, \mathrm{Zu}$ rezitieren: Beiseite! Stop! Zurück, zurück! Weg, weg! ${ }^{67^{73}}$.

Das Pseudopartizip kommt somit nur mit Negation vor, wenn das Verb semantisch negativ ist, so dass die Aussage der Pseudopartizipien in den Bsp. 53f. logisch positiv ist (duplex negatio affirmat). Ansonsten wird die $s \underline{d} m=f$ - oder die $s \underline{d m} . n=f$-Form verwendet.

\footnotetext{
${ }^{71}$ Pyr. 1429a-e (Spr. 566, P).

${ }^{72}$ Pyr. 1742d (Spr. 615, M).

${ }^{73}$ Pyr. 687a-b (Spr. 391, T). Die Übersetzung „Lie down!"von Faulkner, The Ancient Egyptian Pyramid Texts (wie Anm. 16), 129, trägt dem perfektischen Aspekt zu wenig Rechnung; ebenso: K. Sethe, Übersetzung und Kommentar zu den altägyptischen Pyramidentexten, 6 Bde, Glückstadt etc. 1935-1962, Bd. 3, 257: „Auf deine Seite! Du sollst dich niederlegen. Entschlüpfe, entschlüpfe. Von hinnen, von hinnen!“
}

56. $h m=j n y \quad r h=f$, ,Bin ich unkundig, so ist er nicht wissend ${ }^{4{ }^{74}}$.

57. $n(j) r d(j) . n R^{r} w 3 h=f s w r t 3$, ,Re erlaubt nicht, dass er sich auf den Boden legt ${ }^{675}$.

Interessant ist in diesem Zusammenhang der folgende Satz - es handelt sich um einen Ausspruch, der einem Arbeiter bei der Flachsernte beigeschrieben ist -, der einen ironischen Unterton aufweist:

58. $h m . w(j) n m n j m h(3) w . t=f$, „Wir kennen keinen, der bei seiner Arbeit gestorben wäre ${ }^{c c^{76}}$.

Der Unterton („Wir wissen, dass welche fast gestorben sind") wird bewirkt durch die Verwendung des Verbs hlm, "unwissend sein", dessen Bedeutung negativ ist, aber in konträrer Funktion verwendet werden kann ${ }^{77}$. Der Ausdruck „unwissend sein, dass“ entspricht „wissend sein, dass nicht": „Wir sind unwissend, dass einer gestorben ist" impliziert „Wir wissen, dass einer nicht gestorben ist", wobei „nicht gestorben“ "hier ,nicht unbedingt gestorben" ist, d. h. ,fast gestorben“ bedeutet.

\section{Die angepasste Medialität des Pseudopartizips im Mittelägyptischen}

\subsection{Die angebliche Relikthaftigkeit des mittelägyptischen Pseudopartizips}

Die anerkannte Bedeutung des Pseudopartizips - aktiv-intransitiv und passiv-transitiv wird gemeinhin eingeschränkt: Es bleibe stets relikthaft - ein Rest einiger transitiver Verben, die offensichtlich aktive Bedeutung in narrativer Verwendung hätten ${ }^{78}$. Allerdings sind die Belege

\footnotetext{
${ }^{74}$ Pyr. 244c (Spr. 240, T).

${ }^{75}$ Pyr. 813b (Spr. 439, P).

${ }^{76} \mathrm{~N}$. de G. Davies, The Rock tombs of Deir el Gebrâwi, Bd. 2, London 1902, Taf. 6.

${ }^{\pi}$ Vgl. L. R. Horn, A Natural History of Negation, Chicago/London 1989, 273-361.

${ }^{79}$ A. Gardiner, Egyptian Grammar, London ${ }^{2} 1957$, 237, \311 (rh); Loprieno, Ancient Egyptian (wie Anm. 1), 66; Malaise/Winand, Grammaire (wie Anm. 49), 447, $\int 722$ (rh, jrj, $\left.d d, r d j, j n j\right)$; W. S chenkel, Tübinger Einführung in die klassisch-ägyptische Sprache und Schrift, Tübingen 2005, 226, verzeichnet (erst
} 
dafür weder aktiv-transitiv, noch entsprechen sie der aktiv-kausativen Bedeutung mit direktem Objekt des Altägyptischen und können somit nicht als relikthaft eingestuft werden.

59. $r \underline{d}(j) . k(w) j ~ j w t ~ d 3 m . w n$ hwn.w nfr.w $r$ jr(j).t $n=f$ w3.t, „Ich habe veranlasst, dass eine tüchtige Jungmannschaft kam, um ihm den Weg zu bereiten “"

60. $\underline{d} d . k(w) j n(j) r h=j s w$, "Ich aber habe erwidert: Ich kenne ihn nicht ${ }^{480}$.

61. $j n(j) . k(w) j j m r$ 3.t wr.t, ,Ich ließ Unmengen davon (dem zuvor genannten Amethyst) gebracht werden ${ }^{881}$.

62. jr(j).kwj $m$ ck, „Ich habe (gehandelt) mich verhalten/mich bewährt als einer, der eintritt $[\ldots]^{66^{82}}$.

63. jb.kwj $w 3 w p w n w 3 \underline{d} w r$, „Ich war zum Schluss gekommen: Es ist eine Meereswoge ${ }^{\text {c833 }}$.

64. $r d j . k(w) j r$ pr s3 nswt, „Ich befand mich beim Haus eines Königssohns" ${ }^{\text {“4t }}$.

In den Beispielen 59f. folgt dem Pseudopartizip nicht eine Nominalphrase als Objekt, sondern ein Subjunktiv nach $r \underline{d} j$, ,veranlassen, dass “ (Bsp. 59, vgl. Abschnitt 1.2 zu Bsp. 25) resp. eine negierte Aussage nach $\underline{d} d$, ,sagen“ (Bsp. 60). Auch Bsp. 61 bis 64 stellen keine Ausnahme zur Regel dar, da die verwendeten Verben zwar meist transitiv sind, hier jedoch intransitiv verwendet werden und also im Pseudopartizip der Regel entsprechend aktive Bedeutung haben: $j n j$ jm, „davon (partitiv) bringen" (neben transitivem „etwas bringen“), $j b$, ,zum Schluss gekommen sein/glauben, dass" (neben transitivem „etw. wünschen/denken“), $r \underline{d j}$, „, sich begeben haben/sich befinden" (neben transitivem „etw. geben"), $j r j$, „(richtig) gehandelt/sich (richtig)

in dieser Auflage) diesen Gebrauch ,bei besonderer Anteilnahme des Agens am Geschehen, namentlich bei Verben der geistigen Betätigung“.

${ }^{79}$ P. E. Newberry, El Bersheh, London 1894 1895, Bd. 1, Taf. 14, Kol. 2 f.

${ }^{80}$ Sinuhe B 114.

${ }^{81}$ A. I. Sadek, The Amethyst Mining Inscriptions of Wadi el-Hudi, Warminster 1981, Bd. 1, 33, Nr. 14, Z. 10.

${ }^{82}$ Inschrift des $W p$-w3.wt- 3 aus Abydos, München, Glyptothek, WAF Nr. 35 (alt Nr. 40): W. Spiegelberg, Aegyptische Grabsteine und Denksteine aus süddeutschen Sammlungen, Bd. 2, Strassburg 1904, 2-7; Taf. 2, Nr. 3, Z. 16.

${ }^{83}$ Schiffbrüchiger $57 \mathrm{f}$.

${ }^{84}$ Sinuhe B 286. verhalten/sich bewährt haben" (neben transitivem ,etw. machen").

Der folgende syntaktisch aktiv-transitive Satz ist semantisch reflexiv; das ist der Grund für die Verwendung des Pseudopartizips, nicht dass der Stelenbesitzer versucht hätte, einen literarischen Stil zu schreiben ${ }^{85}$.

65. $w d(j) . k w j ~ m=j \quad r \quad b w \underline{h} r n \underline{n t} \quad W s j r$, „Ich setzte meinen Namen an den Kultort des Gottes Osiris“ $\rightarrow$ „Ich verewigte mich am Kultort des Gottes Osiris" ".86.

Die Pseudopartizipien in Bsp. 66f. sind in der pseudoverbalen Konstruktion, außerhalb der 1. Person und mit dem Verb $r h$ verwendet und von daher nicht ganz mit den übrigen Bsp. dieser Gruppe vergleichbar.

66. $j w h m . t=j$ rh.tj $n t \underline{t r} r=f$, „Meine Majestät weiß, dass er (Amun) göttlich ist" ${ }^{87}$.

67. $j w=f r h(. w)$ ts tp $h s k(. w)$, „Er kann einen abgeschnittenen Kopf wieder ansetzen", $j w=f$ $r h(. w) r d(j) . t s m m 3 j h r-s\}=f[\ldots]$, ,er kann einen Löwen hinter sich her gehen lassen $[\ldots]^{\star}, j w=f$ $r h(. w) \underline{t} n w$ jp.wt [...], „er kennt die Zahl der Schreine $[\ldots]^{66^{88}}$.

Dem Pseudopartizip folgt ein Objektssatz (Bsp. 66) resp. ein Infinitiv (Bsp. 67a-b). Bsp. $67 c$ hat ein Objekt nach sich, entspricht aber darin der reflexiv-kausativen Besonderheit von $r h$ im Pseudopartizip, wie sie bereits im Altägyptischen vorkommt (Abschnitt 1.2 mit Bsp. 38). Bsp. 67a-b haben statt eines Nomens („eine Tatsache wissen") ein Verbalnomen bei sich („wissen wie zu tun / tun können"), wobei rh als „semi-auxiliary“ zu bezeichnen ist ${ }^{89}$.

${ }^{85}$ R. Hannig, Pseudopartizip und $s \underline{d} m . n=f$. Der Kernbereich des mittelägyptischen Verbalsystems II (HÄB 32), Hildesheim 1991, 105.

${ }^{86}$ E. A. Wallis Budge (Hg.), Hieroglyphic Texts from Egyptian Stelae \&xc., in the British Museum, Bd. 2, London 1912, Taf. 9, Z. $4 \mathrm{f}$.

${ }^{87}$ Urk. IV, 363, 6.

${ }^{80}$ Pap. Westcar, $7,4$.

${ }^{89} \mathrm{~S}$. Uljas, On Interclausal Relations in Middle Egyptian Object Complementation, in: S. Bickel/ A. Loprieno ( $\mathrm{Hg}$.), Basel Egyptology Prize 1. Junior Research in Egyptian History, Archaeology, and Philology (AH 17), Basel 2003, 393f. 


\subsection{Modalisierung}

Für die aktiv-intransitive Verwendung ist in der 2. und 3. Person deontischer, in der 1. Person dagegen epistemischer Modus festzustellen.

Zunächst sollen die modalen (deontischen) Verwendungen mit der 2. und 3. Person betrachtet werden, die schon im Altägyptischen belegt sind (Bsp. 2f., 6, 10-14, 31, 36, 42, 49, 55).

68. $s 33 . t j ~ h r$ sp $n$ mh.t-jb, „Mögest du dich gehütet haben vor einem (Fall) Vorkommen von Vergesslichkeit" $\rightarrow$ „Hüte dich vor Vergesslichkeit! “"

69. $j j(j) . t j n=j$, ,Mögest du mir gekommen sein“ $\rightarrow$ „Sei mir willkommen! ${ }^{a 91}$.

Im Gegensatz zum Altägyptischen erscheinen im Mittelägyptischen besonders häufig Beispiele, in denen der pseudopartizipiale Wunsch als Eulogie eingeschoben ist.

70. $s w d 3-j b p w n n b=j$ ' $n h(. w) w d z(. w) s n b(. w) h r[\ldots]$, „Dies ist eine Mitteilung an meinen Herrn - er lebe, sei heil und gesund! - betreffend [... $]^{\alpha{ }^{32}}$

71. s3.t nswt Nfrw-R ${ }^{r}{ }^{\text {r } h . t . t j, ~,[K o m m e n ~ d e s ~ . . ., ~ d e s ~}$ großen Vermögensverwalters der] Königstochter Prinzessin Nefrure - sie lebe! - [Senenmut ...] $]^{6 .}$.

Mit der Perfektivität nimmt der Sprecher die Handlung des Adressaten vorweg und erzielt eine eindringliche Mahnung. „Mögest du dich gehütet haben“ bedeutet „Mögest du dich unbedingt hüten“, d. h. „Hüte dich!“ (Bsp. 68) oder eine vorbehaltlose Bitte: „Sei mir gekommen!“ bedeutet „Sei mir ganz willkommen!“ (Bsp. 69) oder einen generellen Wunsch: „Er sei stets mit Leben begabt, heil und gesund!“ (Bsp. 70f.). Die deutsche Übersetzung tendiert im Falle der Mahnung zu Recht zu einer imperativischen Übersetzung. Auch im Ägyptischen ist der

\footnotetext{
${ }^{90}$ Ptahhotep 154 (L2).

${ }^{91}$ Urk. IV, 611, 15.

${ }^{92}$ Pap. Kahun 3.2, Kol. 1f.: F. L. Griffith, Hieratic Papyri from Kahun and Gurob, London 1898, Taf. 27.

${ }_{93}$ Urk. IV, 396, 16.

${ }^{94}$ Die Perfektivität von ' $n h$ im Pseudopartizip lässt sich im Deutschen schlecht ausdrücken: „er möge belebt sein und von nun an leben“ ist mit einfachem „er lebe!" wiederzugeben.
}

Wechsel zwischen diesen beiden deontischen Modalformen der 2. Person festzustellen ${ }^{95}$.

Zur Veranschaulichung des Gebrauchs der 1. Person, der als nächstes betrachtet werden soll, sei zunächst auf das folgende Bsp. verwiesen.

56. $j n(j) . k(w) j j m r$ c $3 . t$ wr.t, ,Ich ließ Unmengen davon (dem zuvor genannten Amethyst) gebracht werden".

Der Text stammt aus der Zeit Sesostris' I. und verwendet das Pseudopartizip noch in der Art des Altägyptischen (vgl. Abschnitt $1.2 \mathrm{zu}$ Bsp. 26-29), hier jedoch ohne Nennung des Patiens der kausativierten Handlung.

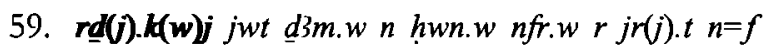
$w 3 . t$, „Ich habe veranlasst, dass eine tüchtige Jungmannschaft kam, um ihm den Weg zu bereiten".

60. $\underline{d d . k(k) j} n(j) r h=j s w$, ,Ich aber habe erwidert: Ich kenne ihn nicht".

62. $j$ r(j). $k w j ~ m ~ k$, ,Ich habe (gehandelt) mich verhalten/mich bewährt als einer, der eintritt $[\ldots]^{\prime \prime}$.

63. jb.kwj $w 3 w p w n w 3 d w r$, „Ich glaubte: Es ist eine Meereswoge".

64. $r d j . k(w) j \quad r$ pr $s 3$ nswt, „Ich befand mich unvermittelt beim Haus eines Königssohns".

72. $j$ r(j).k(wj) $m j$ wd.t.n $n b . t h m=f$, ,Ich nun handelte entsprechend all dem, was seine Majestät angeordnet hatte ${ }^{\left(c^{9} \%\right.}$.

In einem Fall wie Bsp. 72 ist die faktitivresultative Aktionsart vielleicht nicht unmittelbar einsichtig ${ }^{97}$, aber durchaus plausibel: Nach dem Zitat des Befehls des Königs stellt der Sprecher fest: „Ich handelte Punkt für Punkt in der Art und Weise, dass das Resultat dem Befehl seiner Majestät entsprach".

Der Gebrauch in der 1. Person, wie er in Bsp. 59f., 62-64 und 72 vorkommt, kann als Merkmal besonders authentischer Erzählweise von persönlich Erlebtem aufgefasst werden. Der

${ }^{95}$ Z. B. Ptahhotep 300: „Hüte dich (P: ' $h 3$ t $(w)$, L1: $s 3(w) \underline{t}(w)$, L2 mit Pseudopartizip: s33.tj) vor der Habgier!“". Vgl. zum Altägyptischen Edel, Altägyptische Grammatik (wie Anm. 10), 286, § 592.

${ }^{96}$ Stele des Ichernofret, Z. 10: H. Schäfer, Die Mysterien des Osiris in Abydos unter König Sesostris III nach dem Denkstein des Oberschatzmeisters I-chernofret im Berliner Museum, Hildesheim 1964.

${ }^{97}$ Malaise/Winand, Grammaire (wie Anm. 49), $448, \$ 723$. 
Sprecher blickt - zuweilen nicht ohne Selbstbewunderung oder Erstaunen - auf die abgeschlossene Handlung zurück („Es war tatsächlich so ..."). Insofern ist die Verwendung des Pseudopartizips als eine authentifizierende, assertive Sprechhaltung zu verstehen („Ich habe tatsächlich gesagt" vs. „Ich sagte“ $)^{98}$ und dem epistemischen Modus zuzuordnen. Dieser ergibt sich aus dem jeweiligen Kontext, lässt sich aber durch die allgemeinsprachwissenschaftliche Beobachtung untermauern, dass epistemische Modalität, diachronisch gesehen, auf deontische folgt. Die Entwicklung von deontischer zu epistemischer Modalität ist die Folge von subjectivization of meaning". Die indirekt-kausative und somit deontische Bedeutung der altägyptischen Pseudopartizipien der 1. Person (siehe Abschnitt $1.2 \mathrm{zu}$ Bsp. 26-29) hat sich im Mittelägyptischen zu epistemischer Modalität „subjektiviert“. Zu den semantischen Feldern der verwendeten Verben siehe Abschnitt 4.

In Bsp. 60 ist der Sprecher Sinuhe. Dem Fürsten von Retjenu gegenüber leugnet er, den Starken zu kennen. Hier ist wohl mit epistemischem Unterton gemeint: „Ich behauptete selbstverständlich: Ich kenne ihn nicht." Auch im folgenden Beispiel kommentiert Sinuhe mit dieser Ausdrucksweise sein eigenes cleveres Verhalten mit Schmunzeln:

73. $\underline{d} d . k(w) j r=j n=f w s ̌ b=j n=f$, ,Ich sagte ihm (und) antwortete ihm: $[\ldots]$ “ $\rightarrow$ „Ich entgegnete ihm natürlich folgendermaßen: $[\ldots]^{c c^{100}}$.

Es folgt die Antwort, die Sinuhe auf Amunenschis vorhergehende ironische Frage ${ }^{101}$ nach dem Zustand Ägyptens gibt. Sinuhe fühlt sich genötigt, den Regierungsantritt des neuen Königs zu bestätigen, lässt aber eine lange Lobrede auf diesen folgen. Damit pariert er die Frage

\footnotetext{
${ }^{98}$ Das entspräche der "gespannten Haltung" in der „besprochenen Welt" in der Sprechhaltungstheorie Weinrichs ( $\mathrm{H}$. Wein rich, Tempus. Besprochene und erzählte Welt, Stuttgart 1964, 44 -69). - Vgl. auch Anm. 78 (Schenkel).

Siehe Frawley, Linguistic Semantics (wie Anm. 39), 428-430.

${ }_{100}$ Sinuhe B 45 f. - AOS und DM4 schreiben $\underline{d} d . n=f$ $n=j$.

${ }^{101}$ Siehe Jenni, „Sagen zu“ im Ägyptischen (wie Anm. 10), $246 f$.
}

seines Gegenübers, die ihm in seiner Situation als verfänglich erscheint. Die Präposition $r$ mit koreferentiellem Pronomen verstärkt diesen Unterton $^{102}$. Ansonsten steht eine $s d m . n=f$-Form:

74. $d d . n=j$ swt $m j w-m s$, „Ich sagte aber nur die halbe Wahrheit ${ }^{c{ }^{103}}$.

75. $\underline{d}$ d. $n=j n n \underline{d}$ d.t. $n=j m m 3^{r} . t$ nn wn.t grg jm, „Ich sagte das, was ich gesagt hatte, als Wahrheit; ohne dass Lüge dabei war ${ }^{\text {(6104 }}$.

76. $\underline{d} d . n=j n n n m d . w t r r \underline{d}(j) . t r h=t n$ [...], ,Ich sagte dies, um euch kundzutun $[. . .]^{66^{105}}$.

Das Verb $\underline{d} d$ kommt nur im Mittelägyptischen im Pseudopartizip vor, nicht nur in der 1. Person (Bsp. 60 und 73), sondern auch in der 3. Person. Entsprechend der medialen Diathese bedeutet $\underline{d} d$ nicht einfach „sagen/sprechen“, sondern reziprokes Sprechen, d. h. „erwidern“" (vgl. Abschnitt 4 zur Semantik der medialen Diathese). Die erwähnten Stellen mit der 1. Person stammen aus Dialogpartien der Erzählung des Sinuhe. Die drei Beispiele mit der 3. Person (Bsp. 77-79), sind Briefanfänge, die auf ein vorgängiges Schreiben des Adressaten antworten:

77. b3k 'h S3-nh.t $\underline{d d}(. w)$ [..], „Der Palastdiener Sinuhe erwidert: $[\ldots]^{\varsigma 106}$.

78. $s 3 \underline{d} d(. w) n m w . t=f[\ldots]$, „Der Sohn erwidert seiner Mutter $[\ldots]^{\text {s107 }^{107}}$.

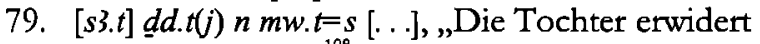
ihrer Mutter $[\ldots]^{\star c^{108}}$.

Die oben zu Bsp. 63 gegebene Übersetzung diente der Verdeutlichung der Satzstruktur, ist aber natürlich nicht kontextgemäß. Der Schiffbrüchige erzählt - auch hier sich selbst kommentierend -, dass er, nach seinem erlittenen Schiffbruch, bei Donnergrollen sofort an unruhiges Meer denkt: „Ich glaubte natürlich, es sei

${ }^{102}$ Vgl. Jenni, Die pronominalen Erweiterungen (wie Anm. 17), $119 \mathrm{f}$

${ }^{103}$ Sinuhe B 37.

104 DZA 23748560 (http://aaew.bbaw.de/dza/index.html).

105 DZA 31731190 (http://aaew.bbaw.de/dza/index.html).

${ }^{106}$ Sinuhe B 204f.

${ }^{107}$ J. P. Allen, The Heqanakht Papyri, New York 2002, Taf. 30: Brief II, recto Kol. 1. So auch Brief III, recto Kol. 1; dagegen Brief I, recto Kol. 1: $r d d$ jn hm-k3 $H k 3-n h t(. w)[\ldots]$.

${ }^{108}$ Allen, The Heqanakht Papyri (wie Anm. 107), Taf. 38: Brief IV, Kol. 1. 
eine Meereswoge". In Tat und Wahrheit ist die akustische Wahrnehmung aber mit dem Erscheinen des Schlangengottes verbunden.

Bsp. 64 stammt von der Passage, in welcher Sinuhe schildert, wie er nach seiner Rückkehr an den ägyptischen Hof und dem Empfang durch die Königsfamilie von den Königskindern hinausgeleitet wird. Gedanklich ist er immer noch in der Audienzhalle und findet sich - er weiß nicht wie - unvermittelt an einem anderen staunenswerten Ort, der mit allem Luxus versehenen Residenz eines Prinzen: „Ich musste mich zu einer Prinzenresidenz begeben haben / Ich hatte mich wohl zu einer Prinzenresidenz begeben" $\rightarrow$ „Ich befand mich plötzlich bei einer Prinzenresidenz / Ich fand mich plötzlich bei einer Prinzenresidenz wieder". Dasselbe Verb ist auch im folgenden Bsp. verwendet:

80. $\lceil h r, n=j r \underline{d}(j) . k w j r j w$ jn $w\} w n w 3 \underline{d} w r$, „Dann fand ich mich an Land (gegeben) gespült von der Meeresbrandung ${ }^{\mathrm{ci}}{ }^{109}$.

Bsp. 80 stellt den seltenen Fall eines Pseudopartizips mit Agensangabe dar, eines Passivs, dem wie der passiven $s \underline{d m} . w=f$-Form und dem tw-Passiv die Angabe des Agens mittels jn + Nominalphrase zugefügt sein kann. Das faktitivresultative Pseudopartizip ist in Bsp. 80 gewählt, um den erreichten Zustand auszudrücken. Mit diesem Satz ist die Schilderung des eigentlichen Schiffbruchs zu Ende; es folgt die Beschreibung dessen, was der Protagonist auf der Insel vorfindet. Auch hier ist die durch jn eingeführte Größe kontextuell gegeben (vgl. Abschnitt 1.1).

\section{Exkurs: passive Konstruktion mit Angabe des Agens vs. aktive Konstruktion}

In Abschnitt 1.1 wurde festgestellt, dass ein bei einem Pseudopartizip vorhandenes und durch $j n$ eingeführtes Agens hohe salience aufweist, dass ein Agens aber mit der Suffixkonjugation zusammen verwendet wird, wenn es eine kontextuell neue Größe einführt, d.h. rhematisch (und nicht thematisch) ist. Es ist im $\mathrm{Zu}$ sammenhang mit der Diskussion der Diathese

\footnotetext{
${ }^{109}$ Schiffbrüchiger 39-41.
}

generell zu fragen, weshalb in gewissen Fällen überhaupt eine passive Konstruktion und nicht eine aktive gewählt wird - Lehrbücher und Grammatiken schweigen sich darüber meist aus. Die Antwort ist im Bereich der Pragmatik zu suchen - ähnlich wie im Deutschen ${ }^{110}$.

Wird ein Satz passiv formuliert mit Angabe eines rhematischen Agens, d. h. mit einer passiven Form der Suffixkonjugation, so hat nicht nur das Prädikat (die passive Verbalform), sondern das Prädikat mit dem Agens zusammen rhematische Funktion („Der Brief wird von dir geschrieben") - im Gegensatz zu dem entsprechenden aktiv formulierten Satz, wo das Agens (Subjekt) thematisch und das Prädikat thematisch ist (,Du schreibst den Brief"). Das Agens ist dabei nicht isoliert hervorgehoben, $d$. h. fokussiert $(, D u$ bist es, von dem der Brief geschrieben wird" "Von dir ist es, dass der Brief geschrieben wird"). Die passive Konstruktion mit Angabe des Agens erlaubt es, über eine kontextuell gegebene Größe (Subjekt/Thema) eine Aussage mit hohem Neuigkeitsgrad zu machen, die das Agens mitberücksichtigt. Dass die Partikel $j n$ in diesem Fall nicht (wie in ihrer fokussierenden Funktion) proklitische Stellung hat, versteht sich von daher von selbst.

81. [...] $r \underline{d}(j) \cdot n=f j r(j) . t(w) n(=j)$ js $m$ jmnt.t jn hlmw.t n.t $h m=f,,[\ldots]$ nachdem er veranlasst hatte, dass mir ein Grab angefertigt werde im Westen durch die Handwerkerschaft seiner Majestät $t^{\text {sil1 }^{11}}$.

Von der Nekropole ist in diesem Beispiel im vorhergehenden Satz die Rede. Für den Stelenbesitzer ist von Bedeutung, dass er in den Genuss eines von Arbeitern des Königs errichteten Grabes kommt.

${ }^{110}$ Vgl. G. Schoenthal, Kontextsemantische Analysen zum Passivgebrauch im heutigen Deutsch. Zur Mitteilungsperspektive im Passivsatz, in: Centre de Recherche en Linguistique Germanique (Nice) ( $\mathrm{Hg}$ ), Das Passiv im Deutschen (Linguistische Arbeiten, Bd. 183), Tübingen 1987, 161-179. - Zum Ägyptischen vgl. Malaise/Winand, Grammaire (wie Anm. 49), 413, \$659.

"Stele des Sarenput Nr. 10, Z. 17 f.: D. Franke, Das Heiligtum des Heqaib auf Elephantine (SAGA 9), Heidelberg 1994, 177-189; Taf. 3. 


\section{Typologische Entwicklung und Semantik des ägyptischen Pseudopartizips}

Für das altägyptische Pseudopartizip ergeben sich deutliche Merkmale der medialen Diathese, bei welcher der Verbalinhalt das Subjekt oder seine Interessen betrifft: Das Agens ist marginalisiert, kausative und direkte Objekte sind reflexiv und die salience ist allgemein hoch. Zu letzterer ist auch die Neutralisierung der Polarität (ohne Möglichkeit semantischer Negierung) zu rechnen.

Die Hierarchie der salience erklärt, dass nichtpronominale Subjekte beim altägyptischen Pseudopartizip verhältnismäßig wenig zahlreich sind, da Nomina in dieser Skala weiter unten stehen als Pronomina. Die Hierarchie von der 1. über die 2. zur 3. Person spiegelt sich wider in der morphologischen Markierung des Pseudopartizips, die in der 3. Person sehr schwach ist (Endung $-a$, markiert durch -.w oder -..$)^{112}$. Das Patiens verhält sich in der Hierarchie der Animiertheit in Relation zur Hierarchie der Atelizität resp. Nonagentivität des Verbs. So finden sich Personen und ihre Körperteile am oberen, Dinge am unteren Ende dieser Skala.

Im Vergleich zum Altägyptischen verliert das Pseudopartizip im Mittelägyptischen die Möglichkeit der transitiv-kausativen Verwendung mit einem agentivischen Verb mit semantisch marginalisiertem Agens von hoher salience (1. Person) und mit direktem Objekt von hoher salience (Fall $\mathrm{d}$ in der Liste zu Beginn des Abschnitts 1.3). Agentivische Verben in aktiver Bedeutung kommen zwar in der 1. Person noch vor, jedoch nur in intransitiver Verwendung und in epistemischem Modus oder semantisch reflexiv. Wie Bsp. 73 mit Anm. 99 zeigt, wurde das Pseudopartizip in dieser Verwendung mit der Zeit nicht mehr verstanden. Vom Altägyptischen her gesehen, ist sie jedoch kein Relikt.

Das Pseudopartizip existiert im Mittelägyptischen als Perfektiv in resultativer Aktionsart und modaler Ausrichtung weiter, andererseits behält

${ }^{112}$ Vgl. A. Loprieno, Das Verbalsystem im Ägyptischen und im Semitischen. Zur Grundlegung einer Aspekttheorie (GOF IV/17), Wiesbaden 1986, 158. es wesentliche seiner Merkmale (Fälle a bis $\mathrm{c}$ in der Liste zu Beginn des Abschnitts 1.3), und zwar diejenigen, die dem aktiv-passiven System nicht zuwiderlaufen. So fristet das ans aktivpassive System angepasste Pseudopartizip im Mittelägyptischen sozusagen ein unauffälliges mediales Dasein, findet aber seine ansehnliche Spezialisierungsnische in resultativ-modaler Hinsicht.

Die semantische Medialität zeigt sich sowohl fürs alt- wie fürs mittelägyptische Pseudopartizip. Die im Pseudopartizip verwendeten Verben lassen sich semantisch ganz grob wie folgt gruppieren (repräsentative Liste ohne Anspruch auf Vollständigkeit - manche Verben müssten je nach Gebrauch in verschiedenen Gruppen erscheinen; die Zuordnung erfolgt nach der vermuteten Grundbedeutung). Dabei zeigt sich zweierlei: Sie fügen sich weitgehend in die allgemeinsprachlich $\mathrm{zu}$ beobachtenden semantischen Gruppen ein, die mediale Kennzeichen haben $^{113}$, wobei die körperlichen Bereiche ${ }^{114}$ für das Ägyptische hervorzuheben sind. Und die semantischen Gruppen bleiben sich vom Altzum Mittelägyptischen (markiert mit A resp. M) gleich $^{115}$.

- Unpersönlich: jrj.tj es ist zu tun A, nfr.w es ist/geht gut $\mathrm{A}$.

- Reziprokes Ereignis: $3 b h$ vermischt sein $M, j c b$ vereinigt sein $\mathrm{A}, j w n$ vereinigt sein $\mathrm{A}$, wšd begrüßt sein $\mathrm{M}, m n j$ angepflockt/vertäut sein $\mathrm{AM}, \underline{h n m}$ vereinigt sein $\mathrm{M}$, stk eingeführt/eingeliedert sein $\mathrm{M}, s p j$ zusammengebunden/gebaut sein $\mathrm{A}, s^{3} 3$ vereinigt sein $M$, sht geflochten/mit Netz gefangen sein $\mathrm{AM}, s \tilde{s} d$ umgebunden sein $\mathrm{A}, k 3 s$ gebunden sein $\mathrm{A}, t(3) s$ geknüpft sein $\mathrm{A}, d m 3$ zusammengebunden sein $\mathrm{M}, \underline{d} d$ sagen im (Dialog)/erwidern M.

${ }^{113}$ Siehe Kemmer, The Middle Voice (wie Anm. 52), besonders 267-270.

${ }^{114}$ Grundlegende Eigenschaftsverben wie lang, scbwer, hoch sein etc. sind unter die körperlichen Qualitäten eingeordnet worden.

${ }^{15}$ Nur die seltene unpersönliche Verwendung (Bsp. 14 und 46) ist im Mittelägyptischen bisher nicht belegt. Die Liste beruht für die altägyptischen Verben im wesentlichen auf Allen, Inflection of the Verb (wie Anm. 63), und E. Doret, The Narrative Verbal System of Old and Middle Egyptian, Genève 1986, für die mittelägyptischen auf Hannig, Pseudopartizip und $s d m . n=f$ (wie Anm. 85), 31-34. 
- Physikalische Veränderung: psj gekocht sein $\mathrm{M}$, $p \underline{d} \underline{\underline{d}} \underline{d}$ verströmt sein (Geruch) A, $m \underline{h} \underline{h}$ verbrannt sein $\mathrm{M}, \boldsymbol{n t b}$ ausgetrocknet/versengt sein $\mathrm{M}, k b b /$ $k b h$ gekühlt sein AM, $t 3$ erhitzt sein M.

- Vorhandensein, Vollkommenheit: jwj bootlos sein $\mathrm{A}, j n j$ gebracht sein $\mathrm{M}, j t j$ ergriffen sein $\mathrm{M}, w \xi$ leer/ausgefallen sein $M$, wdj $m=$ seinen Namen setzen/sich verewigen $\mathrm{M}, f d k$ zerteilt/ausgerottet sein $\mathrm{M}, m 3 w j$ erneuert sein $\mathrm{A}, m h$ gefüllt sein/vom Stapel gelassen sein (Schiff) AM, nfr vollkommen sein AM, $n \check{s}$ auseinandergebracht/verdrängt sein $\mathrm{M}, r d j$ gegeben sein $\mathrm{AM}, h 3 b$ ausgesandt sein $\mathrm{M}$, $h s k$ abgeschnitten sein $\mathrm{AM}$, htm vernichtet sein $\mathrm{M}$, hwwd wohlhabend sein $\mathrm{M}, \mathrm{kmm}$ vollständig sein $\mathrm{A}$, spj übrig sein $\mathrm{M}, \breve{s}^{\tau}$ abgeschnitten sein $\mathrm{A}, \check{s} w j$ leer sein AM, $s d j$ genommen sein A, twt versammelt/vollkommen sein $M, t m$ vollständig sein A, $d b h$ bedürftig sein bei/bitten $\mathrm{A}$, $\underline{d} n j$ abgedämmt/zurückgehalten sein $\mathrm{A}, d r$ ferngehalten/ entfernt/ausgetilgt sein AM, $d r$ zu Ende sein M.

- Existenz: ' $n h$ lebendig sein AM, wnn geworden sein $\mathrm{AM}, m(w) t$ tot sein $\mathrm{AM}, m s j$ geboren sein $\mathrm{AM}$, hpr entstanden sein AM.

- Ereignisbeginn, Anstoß zum Ereignis: jinj gebracht sein lassen $\mathrm{A}, w 3 j$ sich anschicken $\mathrm{M}$, w3h niedergelegt sein lassen $\mathrm{A}$, wdf sich verzögert haben $\mathrm{M}, m^{3^{r}}$ geführt sein lassen $\mathrm{A}, r d j$ veranlasst haben A, krs begraben sein lassen A, s'nh verpflegt sein lassen A, sh3j hinabgeführt sein lassen $\mathrm{A}, d w 3 j$ früh sein/Morgen geworden sein $\mathrm{M}$.

- Lebensqualität: $3 h$ geisthaft sein $A, w j$ allein sein $\mathrm{AM}, \boldsymbol{m} n$ beständig sein $\mathrm{AM}$, nhn Kind sein $\mathrm{A}$, $n h h(j)$ erneuert sein A, ntrj göttlich sein A, nd geschützt sein $A$, rnpj verjüngt sein $A, s 3 h$ zu einem Geist gemacht sein $A, \underline{d} s r$ heilig sein $A, \underline{d} d j$ dauerhaft sein A.

- Personale, moralische Qualität: $j r j$ (richtig) gehandelt/sich verhalten/bewährt haben $A, j k r$ vorzüglich sein $A,{ }^{3} j$ gross sein $A$, ' $w n$ habgierig sein $M$, w3s mächtig sein $\mathrm{A}$, w3క kräftig sein $\mathrm{A}$, wsr mächtig sein $M, b 3$ mächtig sein $A, b j n$ böse sein $M, p^{c} n$ fähig sein $\mathrm{A}, m^{3}$ gerecht sein $\mathrm{A}$, spd (zahnför$\mathrm{mig} / \mathrm{scharf}$ ) wirkungsvoll sein $\mathrm{A}, \mathrm{shm}$ mächtig sein A, $d w$ schlecht sein $\mathrm{M}$.

- Körperliche Qualität: $3 k$ lahm sein/zugrunde gegangen sein $\mathrm{M}, 3 w j$ lang sein (Schritt) AM, 3hd schwach/ohnmächtig sein $\mathrm{M}, 3 d$ verwest sein $\mathrm{M}$, $j w r$ schwanger sein $\mathrm{AM}, j$ leicht sein $\mathrm{M},{ }^{\mathrm{e}} \underline{d}$ unversehrt sein $M, w 3 d$ frisch sein $A, w r$ groß sein (Fuß, Person) AM, wrd müde sein $M$, wsh weit sein (Schritt) A, wtj alt sein A, wd 3 heil sein AM, $b 3 n$ entschlummert sein $A, b 3 g j$ müde sein $A, f n$ schwach sein $\mathrm{M}, m r$ krank sein AM, $n w$ altersschwach sein M, $n h 3$ zerfurcht (Haut)/schrecklich sein $\mathrm{M}$, $n h t$ stark sein $\mathrm{M}$, rwd fest sein (Hand, Arm, Mund, Glieder) AM, $r s$ erwacht sein $M, h 3 j$ nackt sein $A, h b s$ bekleidet sein $A, h m w$ (manuell) geschickt sein $\mathrm{M}, s b 3 g j$ ermüdet sein $\mathrm{A}$, snb ge- sund sein $\mathrm{M}, s h j$ taub sein $\mathrm{M}, s p$ blind sein $\mathrm{M}, k 3 j$ hoch sein AM, gs gesalbt sein M, gs3 zur Seite geneigt sein $\mathrm{M}, t n j$ alt/gebrechlich sein $\mathrm{M}, d \xi r$ rot sein A, ddf gesträubt sein (Haare)/Gänsehaut haben $\mathrm{M}$.

- Körperbehandlung. 3tp beladen sein $M, j\lceil j$ gewaschen/rein sein $M$, e pr ausgerüstet sein $A$, wt einbalsamiert sein $\mathrm{A}$, wnh bekleidet sein $\mathrm{A}$, wrh gesalbt sein $A, w^{c} b$ gewaschen/rein sein A, hsj mit Abzeichen versehen/ausgezeichnet/gelobt sein AM, $h t m$ versehen sein (mit Speisen/Schmuck) A, $s b 3$ gezüchtigt/belehrt sein $\mathrm{M}$, sbn gekrönt sein $\mathrm{A}$, sspd ausgerüstet sein $\mathrm{M}$, $s d$ gekleidet/geschmückt sein AM, št geschmückt sein A, knm eingehüllt sein A, thj satt getrunken/betrunken sein $\mathrm{M}, t \underline{t} j$ bekleidet sein $\mathrm{M}, d n s$ belastet sein $\mathrm{M}, d b 3$ geschmückt sein $\mathrm{A}$.

- Körperliche Empfindung: jbj durstig sein A, nd 3 verdurstet sein $\mathrm{M}, h k r$ hungrig sein $\mathrm{AM}, s 3 j$ satt sein AM.

- Körperverhalten: jnpj auf dem Bauch liegen A, ${ }^{\top} r k$ gebeugt sein $\mathrm{A}$, ${ }^{\top} h^{\Gamma}$ stehen $\mathrm{AM}, w 3 h$ liegen $\mathrm{M}, p 3 h d$ kopfüber sein A, hmsj sitzen AM, htp sich niedergelassen haben/zufrieden sein AM, $h 3 m$ gebeugt sein $\mathrm{M}, h n j$ sich niedergelassen haben $\mathrm{M}$, shd auf dem Kopf stehen M, sš ausgestreckt sein (Beine)/ausgebreitet sein $M$, sdr sich inaktivieren/liegen AM, ksj gebeugt sein A, $g r$ schweigen $\mathrm{M}, d w n$ ausgestreckt sein (Hände) $\mathrm{M}$.

- Bewegung am Ort: wn geöffnet sein $M$, whs gebrochen (Stein)/ausgerupft (Pflanze) sein A, $b 3 k$ bearbeitet sein $\mathrm{M}$, nd $\boldsymbol{d} r$ geschlagen/geglättet sein (Holz) A, hwsj gestampft (Körner, Ziegel etc.)/gemauert sein AM, $h r$ gefallen sein AM, $s 3 h$ getreten sein (mit den Füßen)/belehnt sein M, $s h r$ gefällt sein A, shhr bestrichen sein $\mathrm{M}, \grave{s} n j$ umkreist sein AM, $k d$ gedreht (Topf)/gebaut sein A, gnh beschlagen sein $\mathrm{M}, d b n$ umrundet sein $\mathrm{A}$.

- Bewegung über Distanz: 3s geeilt/gelaufen sein $\mathrm{M}$, ck eingetreten sein AM, jjj / jwj gekommen/da sein $A M, j^{r} r$ aufgestiegen sein $A M, w d 3$ sich begeben haben $\mathrm{M}$, prj hinausgegangen sein $\mathrm{AM}$, ph erreicht haben $\mathrm{M}, n n j$ weggegangen sein $\mathrm{A}, r w j$ gewichen sein $\mathrm{M}, h 3 j$ hinabgegangen sein $\mathrm{AM}, h r j$ sich entfernt haben $A M, h^{j} j$ erschienen sein $A$, $h n t j$ stromaufwärts gefahren sein $A, h s f$ entgegengegangen sein $\mathrm{A}, h t j$ zurückgewichen sein $\mathrm{A}, h d j$ stromabwärts gefahren sein $\mathrm{A}, s(b) j$ gegangen/vergangen sein AM, skr geflohen sein (?) A, $\$ m(j)$ gegangen sein $\mathrm{AM}$, $t n m$ umhergeirrt sein $\mathrm{M}$, $t h j$ abgewichen sein $\mathrm{M}, t s j$ fortgegangen sein $\mathrm{M}$, $t k n$ sich genähert haben/nahe sein $M, d 3 j$ überquert haben $\mathrm{A}$.

- Innere Wahrnehmung. $j b$ vermutet haben/zum Schluss gekommen sein $\mathrm{M}, r h$ kennen/wissen/ vermögen $\mathrm{AM}, h m$ unwissend sein $\mathrm{AM}$.

- Gefühl: $3 d$ agressiv sein A, $h r j$ erfreulich sein A, $h^{\ulcorner} j / h^{r \tau} w$ sich freuen A, $h b$ in Feststimmung sein 
$\mathrm{M}, h n n \mathrm{froh} /$ freundlich sein $\mathrm{M}, s p t$ unzufrieden sein A, snd sich fürchten AM.

(Das Verb $m r j$, „lieben/wünschen“, scheint vor dem Neuen Reich kein Verb des Gefühls gewesen zu sein ${ }^{116}$. Vgl. das folgende Beispiel mit parallelem hsj.kwj (oben in der Rubrik Körperbehandlung aufgeführt) aus dem Mittleren Reich: prr $=j$ $h s(j) . k w j m$ ch $m r(j) . w=j m$ stp-s3, ,(Natürlich nicht anders) als einer, der im Palast mit Auszeichnung versehen worden war und im Palastinnern $\mathrm{Be}$ liebtheit erlangt hatte, kam ich heraus ${ }^{\text {c } 117^{117}}$, mit einem anderen aus der Ramessidenzeit: ${ }^{k}=j$ h $h \mathrm{sj} . k w j$ $p r w=j m r\{t\} . k w j$, ,Gelobt möge ich hineingehen und geliebt möge ich hinausgehen ${ }^{\text {(118 }}$.)

Im jüngeren Ägyptisch überlebt das Pseudopartizip nur noch in grammatikalisierter Form in der sogenannten pseudoverbalen Konstruktion und benötigt dann keine Personenkennzeichnung mehr; die Endung zeigt schwächstmögliche Markierung, nämlich durchwegs -.Ø oder -.tw. Die Semantik der verwendeten Verben bleibt - ohne dass das hier nachgewiesen werden kann - dieselbe.

Typologisch gesehen sind Eigenschaften des altägyptischen Pseudopartizips wie Medialität, Perfektivität, Besonderheiten der Polarität sowie syntaktisch-morphologische Auswirkungen der salience-Hierarchie der Nominalphrasen - verschieden kombiniert - in (split-)ergativ-absolutivischen Sprachen zu beobachten ${ }^{119}$. Nach Comrie tendieren resultative Konstruktionen zu ergativ-absolutiver Syntax ${ }^{120}$. Dieser Sprachtyp behandelt Subjekt und Patiens gleich, im Unterschied zum Agens, während in den meisten europäischen Sprachen Subjekt und Agens dem Patiens gegenüberstehen (sog. nominativ-akkusativische Sprachen). Sehr viele Sprachen enthalten, auch wenn sie überwiegend nominativ-

$116 \mathrm{Vgl}$. B. Mathieu, La poésie amoureuse de l'Égypte ancienne (BdÉ 115), Le Caire 1996, $170 \mathrm{f}$.

Inschrift des $W p$-w3.wt- 3 aus Abydos; München, Glyptothek WAF Nr. 35 [alt Nr. 40]: Spiegelberg, Aegyptische Grabsteine (wie Anm. 82), 2-7; Taf. 2, Nr. 3, Z. 16.

${ }_{118}$ DZA 24158460 (http://aaew.bbaw.de/dza/ index.html); vgl. auch DZA 24158470, 24158510, 24158520

${ }^{119}$ R. M. W. Dixon, Ergativity (Cambridge Studies in Linguistics, Bd. 69), Cambridge 1994, besonders 70 142 zu Perfektivität, Modus, Negation etc.

${ }^{120}$ B. Comrie, Language Universals and Linguistic Typology. Syntax and Morphology, Oxford 1981, 113. akkusativisch organisiert sind, ergativische Züge. Solche sind auch für das Ägyptische ausgemacht worden, wobei sie, diachronisch gesehen, nur im älteren Ägyptisch belegbar sind ${ }^{121}$. Auch das mediale Pseudopartizip muss in die Diskussion um eine ursprüngliche (split-)ergativische Typologie des Ägyptischen gebührend einbezogen werden.

\section{Résumé}

Le présent article étudie, d'un point de vue sémantique, le pseudoparticipe (parfait ancien) comme forme s'inscrivant dans la voix moyenne. La marginalisation de l'agent ainsi que l'absence d'un bénéfactif en sont les traits caractéristiques. Outre l'usage actif avec des verbes intransitifs et passif avec des verbes transitifs, l'égyptien ancien présente également, à la $1^{\text {erc }}$ personne, un usage actif avec des verbes transitifs. Celui-ci intervient lorsque l'objet direct est sémantiquement réfléchi, ainsi que dans un sens transitif-causatif lorsque l'objet direct se trouve sur une position élevée dans l'hiérarchie de salience. Un cas particulier de cet usage, également à la $2^{\mathrm{eme}}$ et $3^{\text {eme }}$ personnes, est celui du verbe rh, "connaître", d'agentivité faible, qui accepte un objet direct grâce à la notion de causativité réfléchie («se faire être savant de quelque chose»).

En moyen égyptien, la voix moyenne du pseudoparticipe se maintient, mais avec suppression de l'usage transitif-causatif - celui qui est contraire à la diathèse proprement passive. Les seules exceptions à cette évolution sont le verbe $r h$ (voir ci-dessus), ainsi que le cas d'un verbe transitif avec objet direct sémantiquement réfléchi. Tous les autres exemples de la $1^{\text {ere }}$ personne se présentent dans une construction intransitive, de modalité épistémique. Parler pour le moyen égyptien d'un usage non productif n'est donc pas approprié.

Les verbes utilisés au pseudoparticipe se concentrent - en ancien comme en moyen égyptien (et par la suite) - sur des champs sémantiques s'inscrivant dans les caractéristiques typiques de la voix moyenne ainsi que celles-ci ont été établis par la linguistique générale. Il s'agit surtout, pour l'égyptien, des champs sémantiques centrés autour du corps, du mouve-

${ }^{121} \mathrm{Zu}$ Spuren von Ergativität im Ägyptischen siehe Loprieno, Ancient Egyptian (wie Anm. 1), 83f; A. Loprieno, Egyptian Linguistics in the Year 2000, in: Z. Hawass (Hg.), Egyptology at the Dawn of the Twenty-first Century, 3 Bde, Cairo/New York 2003, Bd. 3, 79-84; Jenni, Die pronominalen Erweiterungen (wie Anm. 17). 
ment, de l'émotion, de la cognition interne, de l'existence et des actions inchoatives (voire causatives) et réciproques.

\section{Summary}

In the present article, the middle voice of the old perfective (stative) is verified through semantic investigation. Marginalisation of the agent and the absence of a benefactive are defined as characteristic properties thereof. Besides the active-intransitive and the passive-transitive usages, in Old Egyptian the $1^{\text {si }}$ person old perfective is employed active-transitively with semantically reflexive direct objects and as expressive of a transitive-causative meaning with direct objects of high salience. A special case herein is the use of the verb $r h$, "know" (in all persons), which is of weak agentivity, but which due to its reflexive causativity (,to let one's self be informed of something") accepts a direct object also in the old perfective.

In Middle Egyptian the expression of middle voice is still maintained, but the transitive-causative use of the old perfective disappears due to its character as contrary to properly passive voice. Exceptions to this are the verb $r h$ and the continuing 1 st person use with transitive verbs and reflexive direct objects. It is thus not appropriate to consider the latter usage as a relic in Middle Egyptian, although all other cases of the 1st person are intransitive and express epistemic mode.

In Old and Middle Egyptian (as well as later), the verbs used in the old perfective are restricted to ones that display the typical semantic characteristics of the middle voice - as these have been defined through general linguistic observations. The relevant semantic fields in Egyptian are, above all, the body, movement, emotion, internal cognition and existence, as well as inchoative (including causative) and reciprocal situations. 